\title{
Atomic mass measurements of short-lived nuclides around the doubly-magic ${ }^{208} \mathrm{~Pb}$
}

\author{
C. Weber ${ }^{\mathrm{a}, *, 1,2}$, G. Audi ${ }^{\mathrm{b}}$, D. Beck ${ }^{\mathrm{a}}, \mathrm{K}$. Blaum ${ }^{\mathrm{a}, \mathrm{c}, 3}$, G. Bollen ${ }^{\mathrm{d}}$, \\ F. Herfurth ${ }^{\mathrm{a}, \mathrm{c}}$, A. Kellerbauer ${ }^{\mathrm{a}, 4}$, H.-J. Kluge ${ }^{\mathrm{a}, \mathrm{e}}$, D. Lunney ${ }^{\mathrm{b}}$, \\ and S. Schwarz ${ }^{\mathrm{d}}$ \\ ${ }^{a}$ Gesellschaft für Schwerionenforschung mbH, D-64291 Darmstadt, Germany \\ ${ }^{\mathrm{b}}$ CSNSM-IN2P3/CNRS, Université de Paris-Sud, F-91405 Orsay, France \\ ${ }^{\mathrm{c}}$ CERN, CH-1211 Geneva 23, Switzerland \\ ${ }^{\mathrm{d}}$ NSCL, Michigan State University, East Lansing, MI 48824-1321, USA

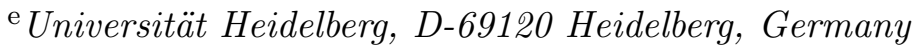

\begin{abstract}
Accurate atomic mass measurements of neutron-deficient and neutron-rich nuclides around the doubly-magic ${ }^{208} \mathrm{~Pb}$ and of neutron-rich cesium isotopes were performed with the Penning trap mass spectrometer ISOLTRAP at ISOLDE/CERN. The masses of ${ }^{145,147} \mathrm{Cs},{ }^{181,183} \mathrm{Tl},{ }^{186} \mathrm{Tl}^{m},{ }^{187} \mathrm{Tl},{ }^{196} \mathrm{Tl}^{m},{ }^{205} \mathrm{Tl},{ }^{197} \mathrm{~Pb}^{m},{ }^{208} \mathrm{~Pb},{ }^{190-197} \mathrm{Bi}$, ${ }^{209,215,216} \mathrm{Bi},{ }^{203,205,229} \mathrm{Fr}$, and ${ }^{214,229,230} \mathrm{Ra}$ were determined. The obtained relative mass uncertainty in the range of $2 \cdot 10^{-7}$ to $2 \cdot 10^{-8}$ is not only required for safe identification of isomeric states but also allows mapping the detailed structure of the mass surface. A mass adjustment procedure was carried out and the results included into the Atomic Mass Evaluation. The resulting separation energies are discussed and the mass spectrometric and laser spectroscopic data are examined for possible correlations.
\end{abstract}

Key words: atomic mass, binding energy, Penning trap, radionuclide, isomer, cesium, thallium, lead, bismuth, radium, francium

PACS: 07.75.+h Mass spectrometers, 21.10.Dr Binding energies and masses, 27.70.+q $150 \leq A \leq 189,27.80 .+\mathrm{w} 190 \leq A \leq 219,27.90 .+\mathrm{b} 220 \leq A$ 


\section{Introduction}

The accurate knowledge of atomic masses is required for many areas of physics [1,2]. In particular, the mass gives access to the nuclear binding energy which in turn reveals distinct features of nuclear structure, notably shell effects and their relative strength as a function of isospin. This paper reports on mass data in the vicinity of the doubly-magic ${ }^{208} \mathrm{~Pb}$ nucleus. Strong nuclear structure effects are observed in particular at the neutron-deficient side from $Z=77$ to $Z=84$. They have been revealed by laser spectroscopic measurements of the optical isotope shift [3,4]. Such measurements allow one to determine the changes of the root-mean-square charge radii $\delta\left\langle r^{2}\right\rangle$ relative to a reference nucleus. Characteristic irregularities were observed in the isotopic chains of iridium $(Z=77)$, platinum $(Z=78)$, gold $(Z=79)$, mercury $(Z=80)$, and thallium $(Z=81)$ at approximately mid-shell $(N=104)$. The most prominent example is the odd-even shape staggering effect within the mercury isotopes [5,6]. These phenomena were first explained for mercury as two different minima in the potential energy surface at a weakly oblate and a stronger prolate deformation (shape coexistence) [7].

In addition, several rotational bands were observed in the nuclear excitation spectra of these nuclei. An overview on nuclear shape coexistence of evenmass nuclides is given in [8]. In ${ }^{186} \mathrm{~Pb}$, even triple shape coexistence has been observed [9]. Here, the three lowest energy states having spin/parity $0^{+}$correspond to spherical, oblate and prolate shapes, all within an energy spanning only $650 \mathrm{keV}$. These phenomena are described by particle-hole excitations of proton pairs across the closed shell [10,11]. The interaction of additional proton-hole pairs and valence neutrons will lead to a minimum in energy around mid-shell. If the energies of the ground state and the intruder state are close, tiny changes in the nuclear binding energy are decisive for the nuclear ground state shape.

Such minute nuclear binding energy effects in this mass range $(A \geq 180)$ are only accessible by mass determinations with mass uncertainties $\delta m$ of $10-20 \mathrm{keV}$. This has been demonstrated by ISOLTRAP measurements on neutron-deficient mercury isotopes [12,13,14]. In that work, isomeric states, a $54-\mathrm{keV}$ level in ${ }^{187} \mathrm{Hg}$ and a $128-\mathrm{keV}$ level in ${ }^{191} \mathrm{Hg}$ were even identified. This was the first example of nuclear spectroscopy by mass spectrometry. The present work complements the information on high-precision masses in this area of the chart of nuclei with data on bismuth, lead, and thallium isotopes.

\footnotetext{
* Corresponding author. Email address: christine.weber@phys.jyu.fi (C. Weber).

1 This publication comprises part of the Ph.D. thesis of C. Weber.

2 Present address: University of Jyväskylä, FIN-40014 Jyväskylä, Finland

3 Present address: Universität Mainz, D-55099 Mainz, Germany

4 Present address: MPI für Kernphysik, D-69029 Heidelberg, Germany
} 
The interconnection of the nuclides studied in this work by $\alpha$-decay chains results in an improvement of further mass values up to $Z \approx 92$.

In case no direct experimental data existed, previous mass values were obtained by a combination of up to four $Q_{\alpha}$ links with results from mass spectrometry. For example, the $\alpha$-decay of neutron-deficient bismuth was studied at the LISOL facility for odd [15] and even isotopes [16]. All direct data being available in this region are either ISOLTRAP measurements [13,17,18] or mass determinations by the Schottky technique at the Experimental Storage Ring ESR [19,20,21]. A detailed comparison to the latter data sets is made in Sec. 6.5. Mass values of neutron-rich cesium isotopes were previously determined by triplet measurements with a Mattauch-Herzog spectrometer [22] and [23]. A particular challenge while addressing the neutron-deficient nuclides experimentally is caused by the existence of two or even three isomeric states with both short half-lives and low excitation energies of sometimes less than $100 \mathrm{keV}$. This requires an extremely high mass resolution of up to $10^{7}$.

Masses on the neutron-rich side of the doubly magic ${ }^{208} \mathrm{~Pb}$ nucleus are of great importance for predictions on the stability of superheavy elements, which are stabilized by shell corrections 24]. A variation of this correction by about $1 \mathrm{MeV}$ leads to changes in the calculated half-lives for spontaneous fission or $\alpha$ decay by several orders of magnitude. Since no experimental mass values are available in this region, model calculations of the shell correction can be verified at the adjacent shell closure of $Z=82$.

Furthermore, accurate mass values are required in order to provide reliable reference masses for the calibration of mass data obtained in measurements at the Experimental Storage Ring ESR [19,20,21. In an ESR mass determination on neutron-deficient nuclides [20,21] 117 reference masses were used in order to calibrate the 466 mass values studied in that experiment. In the neutron-rich region the number of reference masses is particularly low.

\section{Experimental setup and procedure}

The Penning trap mass spectrometer ISOLTRAP [25, 26, 27] is installed at the on-line isotope separator ISOLDE [28] at CERN. Here, mass-separated beams of short-lived nuclides are provided for different experiments at an energy of $60 \mathrm{keV}$. They are produced by bombarding a thick target with high-energy $(1.4 \mathrm{GeV})$ proton pulses $\left(3 \times 10^{13}\right.$ protons/pulse $)$ which induce spallation, fission, or fragmentation reactions. Reaction products diffuse from the heated target into an ion source region. Here, they are ionized either by surface ionization, by electron impact in a hot plasma, or a resonant photo ionization in a resonance ionization laser ion source (RILIS) [29,30].

In this experiment nuclides around the shell closure of $Z=82$ were produced by proton-induced $(1.4-\mathrm{GeV})$ spallation and fragmentation reactions 


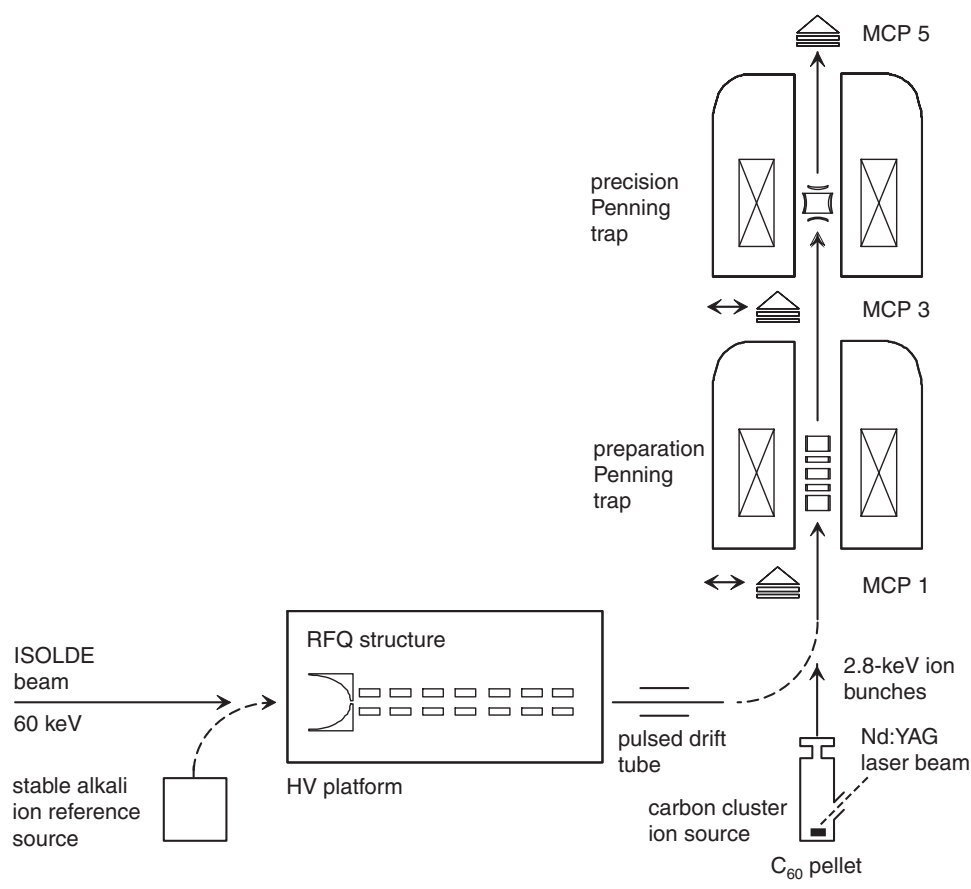

Fig. 1. Schematic view of the Penning trap mass spectrometer ISOLTRAP (For details see text).

on a ${ }^{232} \mathrm{ThC}_{2}$ target $\left(51 \mathrm{~g} / \mathrm{cm}^{2}\right)$. For lead and bismuth a resonant laser ionization was applied, whereas cesium, thallium, francium, and radium were surface-ionized. Finally, the ions were mass-selected with a resolving power of $R=m / \Delta m(\mathrm{FWHM}) \approx 1000$ in the high-resolution separator (HRS).

Figure 1 shows a schematic of the ISOLTRAP mass spectrometer. The radiofrequency quadrupole (RFQ) cooler and buncher [26] decelerates and accumulates the continuous $60-\mathrm{keV}$ ISOLDE beam and delivers a cooled, lowenergy ion bunch for efficient injection into the preparation Penning trap [31]. Here, a mass-selective buffer-gas cooling scheme 32] is employed in order to separate isobars. A resolving power, $R=\nu_{\mathrm{c}} / \Delta \nu_{\mathrm{c}}(\mathrm{FWHM})=m / \Delta m(\mathrm{FWHM})$, of up to $10^{5}$ can be achieved in this process. In the precision Penning trap, the mass $m$ of a stored ion species with charge $q$ is determined via a measurement of its cyclotron frequency $\nu_{\mathrm{c}}=q B /(2 \pi m)$, where $B$ denotes the strength of the magnetic field. A cyclotron resonance spectrum is obtained by the time-offlight detection method [33,34]: The extra radial kinetic energy resulting from the resonant excitation with an azimuthal quadrupolar RF-field at $\nu_{\mathrm{c}}$ is detected by a reduction in the time of flight of the ejected ions towards a detector (MCP5 in Fig. 1). The resolving power is approximately given by the product of the cyclotron frequency and the observation time: $R \approx 1.25 \cdot \nu_{\mathrm{c}} \cdot T_{\text {obs }}[35$ ]. A more detailed description of a typical measurement cycle at ISOLTRAP can be found in a recent publication [36].

An illustrative example of a cyclotron resonance curve is shown in Fig. 2, In such a resonance curve, the mean time of flight of the ions is displayed as a function of the applied radiofrequency, and a resonant excitation can be rec- 


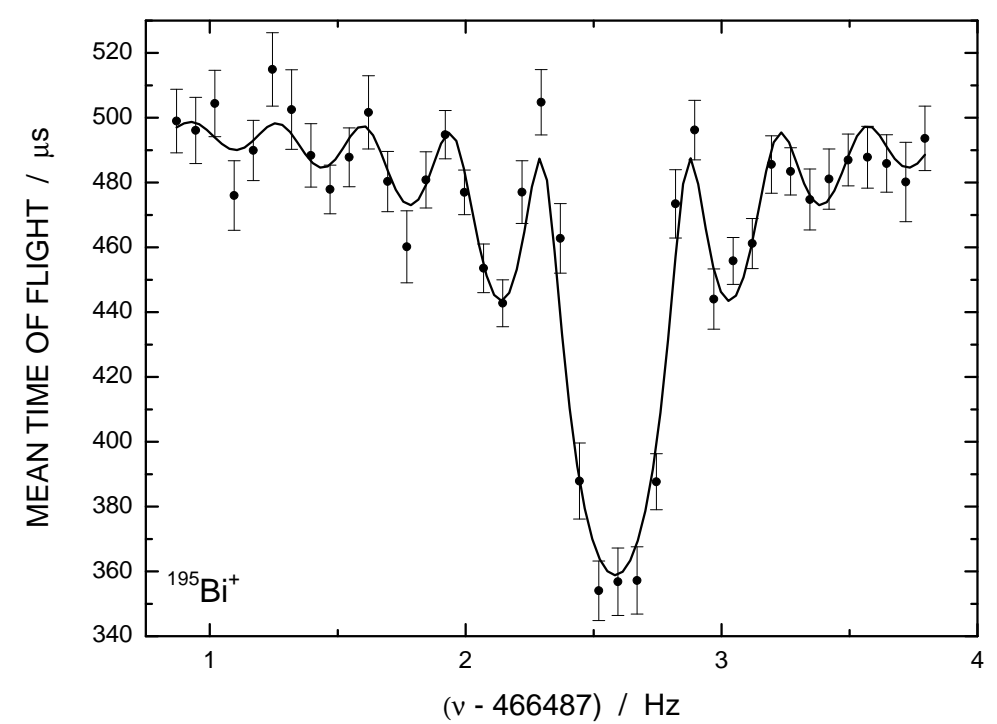

Fig. 2. Cyclotron resonance curve of ${ }^{195} \mathrm{Bi}^{+}$ions. The solid line is a fit of the theoretical lineshape [34] to the data points.

ognized by a shorter flight time. The diagram comprises 16 frequency scans containing a total amount of 1820 detected ions. The linewidth of the central resonance, as given by the Fourier limit $\Delta \nu_{\mathrm{c}}(\mathrm{FWHM}) \approx 1 / T_{\text {obs }}$, results in a resolving power of $R \approx 1.4 \times 10^{6}$ (mass resolution $\Delta m=120 \mathrm{keV}$ ) with an excitation time of the quadrupolar RF-field $T_{\mathrm{obs}}=3 \mathrm{~s}$. This is sufficiently high to resolve ground and isomeric states of ${ }^{195} \mathrm{Bi}$. The theoretical lineshape 34 was used to fit the data points. In order to calibrate the strength of the magnetic field $B$, the cyclotron frequency of a reference nuclide with well-known mass value is determined in regular time intervals. This allows for the accurate mass determination of short-lived nuclides far from stability with relative mass uncertainties $\delta m / m \approx 10^{-8}$ [37,38. Examples of recent measurements on ${ }^{38} \mathrm{Ca}$ [39] as well as on several isotopes of potassium [40], nickel, copper, gallium [41], and krypton [42] cover a wide mass range from $A=35$ to 95 .

\section{Data analysis}

\subsection{Determination of the cyclotron frequency ratios}

The primary result of an ISOLTRAP measurement is the cyclotron frequency ratio $r=\nu_{\mathrm{c}, \mathrm{ref}} / \nu_{\mathrm{c}}$ of a reference ion with well-known mass relative to the ion species to be determined. Thus, these experimental ratios are independent

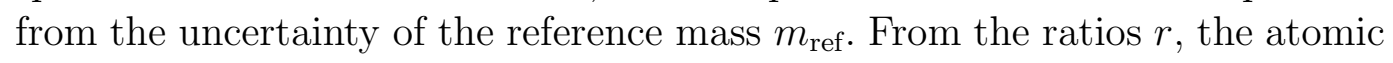


mass of the nuclide under study can be deduced by

$$
m=\frac{\nu_{\mathrm{c}, \mathrm{ref}}}{\nu_{\mathrm{c}}}\left(m_{\mathrm{ref}}-m_{\mathrm{e}}\right)+m_{\mathrm{e}}
$$

Here, the atomic mass of the reference ion is indicated by $m_{\mathrm{ref}}$ and $m_{\mathrm{e}}$ is the mass of the electron. A correction for the binding energy of the missing electron in the order of eV can be neglected.

Extensive studies using carbon cluster ions [37,43] helped to improve the assessment of systematic effects. For example, systematic errors like massdependent frequency shifts or the effect of a variation in the magnetic field strength were quantified. In addition, these investigations result in a better estimate of the final uncertainty of the mass determination. The residual systematic error of the ISOLTRAP mass measurements, estimated previously to be $1 \times 10^{-7}$, was determined in 37] to be

$$
\left(\frac{\delta m}{m}\right)_{\mathrm{res}}=8 \times 10^{-9} \text {. }
$$

The data analysis was performed as described in [37]. Some aspects that are essential in the analysis of the data presented here are summarized in the following.

\subsection{The presence of ions of different mass values}

Simultaneous storage of ions of different mass values may result in a shift of the line center of the cyclotron frequency resonance. Such possible contaminations depend on the ion production process. In the case of measurements at ISOLTRAP, isobars or nuclides in isomeric states might be present in addition to the nuclide under investigation. Systematic experimental studies using 10

to 70 ions stored in the trap as well as simulations with two ions interacting by Coulomb forces are discussed in [44] and [45]: If the two ions have different masses, the resulting frequency shifts depend on the relative distance of the line centers divided by the linewidth that is chosen during the measurement process. If both lines cannot be resolved, only one cyclotron resonance is observed at the frequency of the center of gravity. If the cyclotron resonances of both ion species can be resolved, both line centers are shifted to lower frequencies. The size of the shift has been found to be proportional to the number of stored ions.

To be able to exclude the possible presence of ions of different mass in these measurements, the line center of the cyclotron resonance is analyzed with respect to the number of ions registered by MCP 5 (see Fig. 1) in a so-called count-rate-class analysis. The number of ions counted by the microchannel 

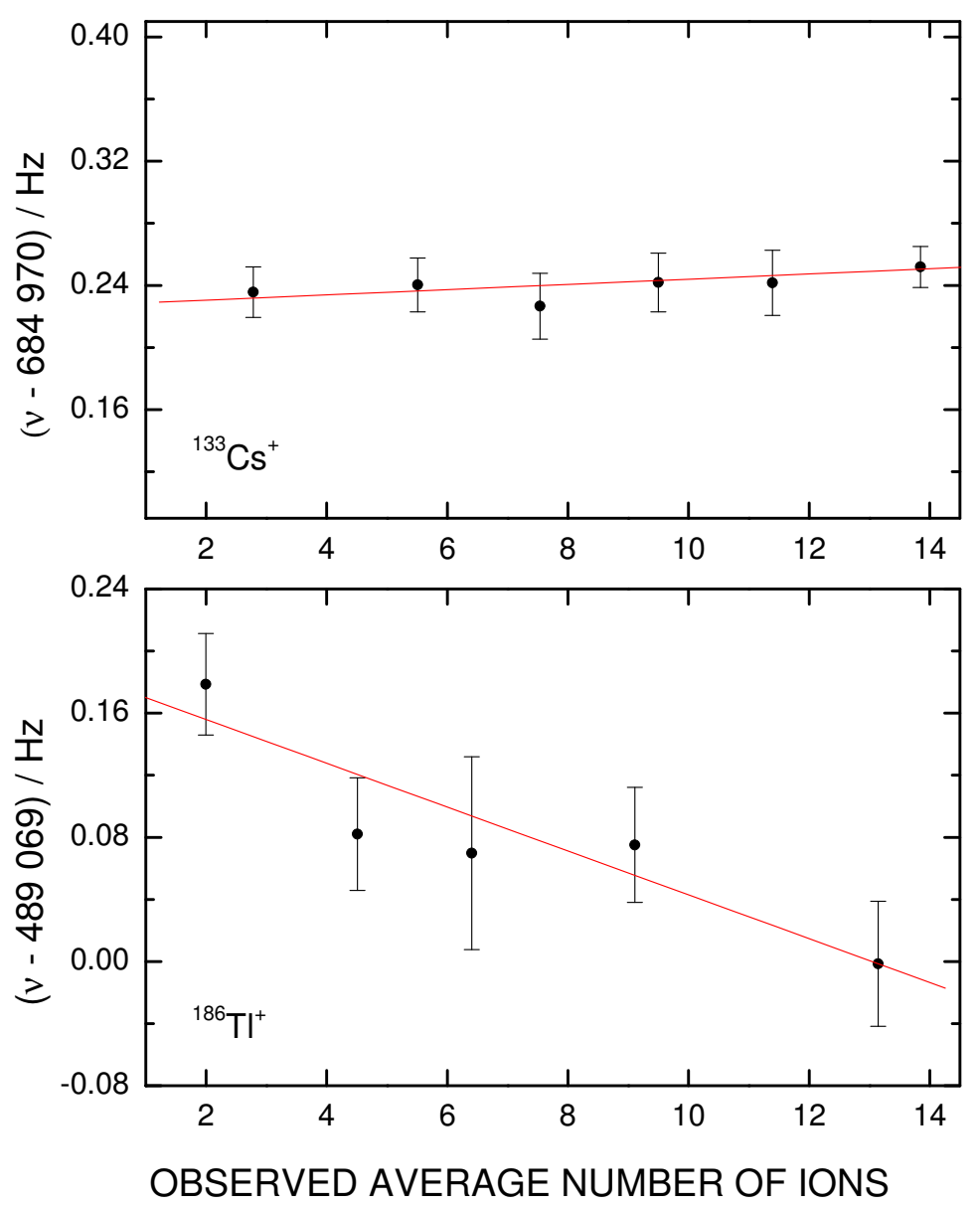

Fig. 3. Dependance of the frequency of the cyclotron resonance on the number of detected ions for ${ }^{133} \mathrm{Cs}$ (top) and ${ }^{186} \mathrm{Tl}$ (bottom). Both measurements were performed with an excitation time $T_{\mathrm{obs}}=1.5 \mathrm{~s}$. The center frequency $\nu$ is plotted versus the count-rate class containing an average number of $N_{\mathrm{av}}=320$ ions as detected by MCP 5 (see Fig. 11). The straight line is a linear regression weighted by the individual error bars. The frequency $\nu_{c}$, extrapolated to zero ions and its respective error is used as the final result.

plate detector after each irradiation of the stored ion cloud with radiofrequency and ejection from the trap is subdivided into at least three count-rate classes with comparable average ion counts $N_{\mathrm{av}}$. A possible shift induced by a contamination is hence corrected by extrapolating the cyclotron frequency to a vanishing number of detected ions. Two examples of a count-rate-class analysis, both with the same average number of ions per count-rate class $N_{\mathrm{av}}$, are shown in Fig. 3. The upper plot shows a measurement done with stable ${ }^{133} \mathrm{Cs}$ ions from the off-line alkali ion source (see Fig. 1), which delivers only surface-ionized alkali elements. The resulting fit of the slope of $2(2) \mathrm{mHz} / \mathrm{ion}$ shows no count-rate dependent effect. The lower one shows a measurement of the radionuclide ${ }^{186} \mathrm{Tl}$ as delivered by ISOLDE. With an increasing number of detected ions per cycle a significant slope of $-14(4) \mathrm{mHz} /$ ion of the cyclotron 
frequency towards lower values is observed. This might be due to the presence of ions in the isomeric state. See also the discussion on the nuclide ${ }^{186} \mathrm{Tl}$ in Sec. 6.2. Combining several measurements, the final value of a frequency ratio is obtained from the weighted mean of individual results.

\subsection{Correcting the mass-dependent systematic frequency shift}

An ion species $q / m$ experiences a frequency shift which can originate for example from electric field imperfections or a possible misalignment of the magnetic and electric field axis versus each other [25]. Since these shifts are to first order mass-independent, this results in a systematic shift in the determination of the frequency ratios, depending on the mass difference $\left(m-m_{\text {ref }}\right)$ between the mass of interest $m$ and the reference mass $m_{\text {ref. }}$ The upper limit of this effect has been previously estimated to $\delta m / m \leq 2 \times 10^{-9} / \mathrm{u}$ [46]. Frequency ratios between carbon clusters whose values are exactly known were quantified by a linear dependance [37,

$$
\frac{r-r_{\mathrm{th}}}{r}=-1.6(4) \cdot 10^{-10} \cdot\left(m-m_{\mathrm{ref}}\right) / \mathrm{u}
$$

where $r-r_{\text {th }}$ is the difference between the measured and the true frequency ratio. In the experiments reported here, covering the mass region between $A=180$ and $A=230$, the reference mass of ${ }^{133} \mathrm{C} \rrbracket(\delta m=25 \mathrm{eV})$ differs by about 70 mass units from the mass to be determined. After applying the correction given by Eq. (3) an uncertainty of the same size,

$$
\frac{\sigma_{m}\left(\nu_{\mathrm{ref}}\right)}{\nu_{\mathrm{ref}}}=1.6 \cdot 10^{-10} \cdot\left(m-m_{\mathrm{ref}}\right) / \mathrm{u}
$$

is added quadratically to the uncertainty. Due to the large mass difference $\left(m-m_{\text {ref }}\right)$, this yields an additional error on the order of $1 \mathrm{keV}$ to the deduced mass excess value. In the end, the residual systematic uncertainty of $8 \times 10^{-9}$ (Eq. (2) ) is added quadratically to obtain the final uncertainty.

\subsection{Mass-to-state assignment}

An unambiguous mass assignment to a specific state, ground state or isomer, is not possible if a non-resolved mixture of nuclei in their ground and isomeric

\footnotetext{
I This reference was chosen due to its high purity when delivered from the ISOLTRAP off-line ion source.
} 
states is present. If the production ratio $R_{\text {ig }}$ (isomer to ground state), and the isomeric excitation energy $E$ are known, the ground state mass $m$ can be extrapolated from the mass $m^{*}$, corresponding to the observed cyclotron resonance, by [23]

$$
m^{*}=m+\frac{R_{\mathrm{ig}}}{R_{\mathrm{ig}}+1} E
$$

If the production ratio of two ion species in ground and isomeric state is not known, the presence of the so-called contamination can be observed in the data analysis by studying the behavior of the resulting cyclotron frequency as a function of the count rate class (see Fig. 3). Only in the rare case of very similar half-lives of the ion under investigation and contaminating ion, contaminations might remain undiscovered.

Table 1 lists all relevant data of the nuclides that have been studied: Halflife $T_{1 / 2}$, spin state $I^{\Pi}$ and the isomeric excitation energy $E$. In case several isomeric states are known to exist, those having a half-life longer than $T_{1 / 2} \geq 500 \mathrm{~ns}$ are listed $\|$. The assignment of a measured mass value to an isomeric state is performed under the assumption that isomeric states with a given $I^{\Pi}$ and excitation energy might appear as listed in Tab. 1. The chosen $\mathrm{RF}$-excitation time results in a width of the cyclotron resonance $\Delta \nu_{\mathrm{c}}(\mathrm{FWHM})$, which corresponds to a width $\Delta m(\mathrm{FWHM}) c^{2}$ in energy units. This value is also called mass resolution and is listed in the last column, since it serves as an important criterion for the observation of a contamination and hence for the identification of the states.

Table 1: Half-lives $T_{1 / 2}$, spin states $I^{\Pi}$ and excitation energies $E$ of the nuclides studied in the present work (from NUBASE [47] and [48]). The full width at half maximum of the cyclotron resonance in energy units $\Delta m c^{2}$ that has been used in individual measurements is listed in the last column. Explanations are given at the end of the table.

\begin{tabular}{lcccc}
\hline Nuclide & Half-Life & Spin/Parity & Excitation & $\Delta m($ FWHM $) c^{2}$ \\
& $T_{1 / 2}$ & $I^{\Pi}$ & energy $E[\mathrm{keV}]$ & {$[\mathrm{keV}]$} \\
\hline${ }^{145} \mathrm{Cs}$ & $582 \mathrm{~ms}$ & $3 / 2^{+}$ & & 220 \\
${ }^{147} \mathrm{Cs}$ & $225 \mathrm{~ms}$ & $\left(3 / 2^{+}\right)$ & 660 \\
${ }^{181} \mathrm{Tl}$ & $3.4 \mathrm{~s}$ & $1 / 2^{+} \#$ & & 340 \\
\hline
\end{tabular}

$\|$ Such short half-lives are also given in order to provide information on spin systematics. Nuclides with $T_{1 / 2} \leq 50 \mathrm{~ms}$ are not considered as contaminants in the studies reported here, since the typical storage time of $1 \mathrm{~s}$ exceeds by far $T_{1 / 2}$. 
Table 1: (Continued).

\begin{tabular}{|c|c|c|c|c|c|c|}
\hline \multirow{2}{*}{$\begin{array}{l}\text { Nuclide } \\
{ }^{181} \mathrm{Tl}^{m}\end{array}$} & & \multicolumn{2}{|c|}{$\begin{array}{c}\text { Half-Life } \\
T_{1 / 2} \\
\end{array}$} & \multirow{2}{*}{$\begin{array}{c}\text { Spin/Parity } \\
\frac{I^{\Pi}}{9 / 2^{-} \#}\end{array}$} & \multirow{2}{*}{$\begin{array}{c}\begin{array}{c}\text { Excitation } \\
\text { energy } E[\mathrm{keV}]\end{array} \\
850(30)\end{array}$} & \multirow{2}{*}{$\begin{array}{c}\Delta m(\mathrm{FWHM}) c^{2} \\
{[\mathrm{keV}]}\end{array}$} \\
\hline & & 2.7 & $\mathrm{~ms}$ & & & \\
\hline${ }^{183} \mathrm{Tl}$ & & 6.9 & $\mathrm{~s}$ & $1 / 2^{+} \#$ & & 340 \\
\hline${ }^{183} \mathrm{Tl}^{m}$ & & 60 & $\mathrm{~ms}$ & $9 / 2^{-} \#$ & $625(17)$ & 340 \\
\hline${ }^{186} \mathrm{Tl}$ & $* \&$ & $40 \#$ & $\mathrm{~s}$ & $\left(2^{-}\right)$ & & 210 \\
\hline${ }^{186} \mathrm{Tl}^{m}$ & $* \&$ & 27.5 & $\mathrm{~s}$ & $\left(7^{+}\right)$ & $40(190)$ & 210 \\
\hline${ }^{186} \mathrm{Tl}^{n}$ & & 2.9 & $\mathrm{~s}$ & $\left(10^{-}\right)$ & $420(190)$ & 210 \\
\hline${ }^{187} \mathrm{Tl}$ & & 51 & $\mathrm{~s}$ & $\left(1 / 2^{+}\right)$ & & 540 \\
\hline${ }^{187} \mathrm{Tl}^{m}$ & & 15.6 & $\mathrm{~s}$ & $\left(9 / 2^{-}\right)$ & $332(4)$ & 540 \\
\hline${ }^{196} \mathrm{Tl}$ & & 1.84 & $\mathrm{~h}$ & $2^{-}$ & & 120 \\
\hline${ }^{196} \mathrm{Tl}^{m}$ & & 1.41 & $\mathrm{~h}$ & $\left(7^{+}\right)$ & $394.2(5)$ & 120 \\
\hline${ }^{205} \mathrm{Tl}$ & & stab & & $1 / 2^{+}$ & & 430 \\
\hline${ }^{197} \mathrm{~Pb}$ & & 8 & $\min$ & $3 / 2^{-}$ & & 120 \\
\hline${ }^{197} \mathrm{~Pb}^{m}$ & & 43 & $\min$ & $13 / 2^{+}$ & $319.3(7)$ & 120 \\
\hline${ }^{208} \mathrm{~Pb}$ & & stab. & & $0^{+}$ & & 270 \\
\hline${ }^{190} \mathrm{Bi}$ & $*$ & 6.3 & $\mathrm{~s}$ & $\left(3^{+}\right)$ & & 560 \\
\hline${ }^{190} \mathrm{Bi}^{m}$ & $*$ & 6.2 & $\mathrm{~s}$ & $\left(10^{-}\right)$ & 150(190) & 560 \\
\hline${ }^{190} \mathrm{Bi}^{n}$ & & $\geq 500$ & ns & $7^{+} \#$ & $420(190)$ & 560 \\
\hline${ }^{191} \mathrm{Bi}$ & & 12.3 & $\mathrm{~s}$ & $\left(9 / 2^{-}\right)$ & & 110 \\
\hline${ }^{191} \mathrm{Bi}^{m}$ & & 128 & $\mathrm{~ms}$ & $\left(1 / 2^{+}\right)$ & $242(7)$ & 110 \\
\hline${ }^{192} \mathrm{Bi}$ & & 34.6 & $\mathrm{~s}$ & $\left(3^{+}\right)$ & & 110 \\
\hline${ }^{192} \mathrm{Bi}^{m}$ & & 39.6 & $\mathrm{~s}$ & $\left(10^{-}\right)$ & $210(50) \#$ & 110 \\
\hline${ }^{193} \mathrm{Bi}$ & & 67 & $\mathrm{~s}$ & $\left(9 / 2^{-}\right)$ & & 110 \\
\hline${ }^{193} \mathrm{Bi}^{m}$ & & 3.2 & $\mathrm{~s}$ & $\left(1 / 2^{+}\right)$ & $308(7)$ & 110 \\
\hline${ }^{194} \mathrm{Bi}$ & $*$ & 95 & $\mathrm{~s}$ & $\left(3^{+}\right)$ & & 580 \\
\hline${ }^{194} \mathrm{Bi}^{m}$ & * & 125 & $\mathrm{~s}$ & $\left(6^{+}, 7^{+}\right)$ & 100(70)\# & 580 \\
\hline${ }^{194} \mathrm{Bi}^{n}$ & $*$ & 115 & $\mathrm{~s}$ & $\left(10^{-}\right)$ & $230(310)$ & 580 \\
\hline
\end{tabular}


Table 1: (Continued).

\begin{tabular}{|c|c|c|c|c|c|}
\hline \multirow{2}{*}{$\begin{array}{l}\text { Nuclide } \\
{ }^{195} \mathrm{Bi}\end{array}$} & \multicolumn{2}{|c|}{$\begin{array}{c}\text { Half-Life } \\
\qquad T_{1 / 2} \\
\end{array}$} & \multirow{2}{*}{$\begin{array}{c}\text { Spin/Parity } \\
\frac{I^{\Pi}}{9 / 2^{-} \#}\end{array}$} & \multirow[t]{2}{*}{$\begin{array}{c}\text { Excitation } \\
\text { energy } E[\mathrm{keV}]\end{array}$} & \multirow{2}{*}{$\begin{array}{c}\Delta m(\mathrm{FWHM}) c^{2} \\
{[\mathrm{keV}]} \\
120\end{array}$} \\
\hline & 183 & s & & & \\
\hline${ }^{195} \mathrm{Bi}^{m}$ & 87 & $\mathrm{~s}$ & $1 / 2^{+} \#$ & $399(6)$ & 120 \\
\hline${ }^{196} \mathrm{Bi}$ & 5.1 & $\min$ & $\left(3^{+}\right)$ & & 590 \\
\hline${ }^{196} \mathrm{Bi}^{m}$ & 0.6 & s & $\left(7^{+}\right)$ & $167(3)$ & 590 \\
\hline${ }^{196} \mathrm{Bi}^{n}$ & 4 & $\min$ & $\left(10^{-}\right)$ & $270(4)$ & 590 \\
\hline${ }^{197} \mathrm{Bi}$ & 9.3 & $\min$ & $\left(9 / 2^{-}\right)$ & & 600 \\
\hline${ }^{197} \mathrm{Bi}^{m}$ & 5.04 & $\min$ & $\left(1 / 2^{+}\right)$ & $350(160)$ & 600 \\
\hline${ }^{209} \mathrm{Bi}$ & 19 & Ey & $9 / 2^{-}$ & & 270 \\
\hline${ }^{215} \mathrm{Bi}$ & 7.6 & $\min$ & $\left(9 / 2^{-}\right)$ & & 470 \\
\hline${ }^{215} \mathrm{Bi}^{m}$ & 36.4 & $\min$ & $\left(25 / 2^{-}\right)$ & $1347.5(2.5)$ & 470 \\
\hline${ }^{216} \mathrm{Bi}$ & 2.17 & $\min$ & $1^{-} \#$ & & 480 \\
\hline${ }^{203} \mathrm{Fr}$ & 550 & ms & $9 / 2^{-} \#$ & & 420 \\
\hline${ }^{205} \mathrm{Fr}$ & 3.85 & $\mathrm{~s}$ & $\left(9 / 2^{-}\right)$ & & 260 \\
\hline${ }^{229} \mathrm{Fr}$ & 50.2 & $\mathrm{~s}$ & $1 / 2^{+} \#$ & & 810 \\
\hline${ }^{214} \mathrm{Ra}$ & 2.46 & s & $0^{+}$ & & 470 \\
\hline${ }^{229} \mathrm{Ra}$ & 4 & $\min$ & $5 / 2^{(+)}$ & & 810 \\
\hline${ }^{230} \mathrm{Ra}$ & 93 & $\min$ & $0^{+}$ & & 330 \\
\hline
\end{tabular}

Isomeric assignment:

* In case the uncertainty $\sigma$ of the excitation energy $E$ is larger than half the energy $(\sigma \geq E / 2)$ an asterisk has been added.

\& In case the ordering of the ground and isomeric states have been reversed compared to ENSDF (Evaluated Nuclear Structure Data Files), an '\&' sign has been added.

Half-life:

\# values estimated from systematic trends in neighboring nuclides with the same $Z$ and $N$ parities (even or odd).

Ey $1 \mathrm{Ey}=10^{18} \mathrm{y}, 1$ exayear.

Spin and parity:

( ) uncertain spin and/ or parity.

\# values estimated from systematic trends in neighboring nuclides with the same $Z$ and $N$ parities (even or odd). 


\subsection{Effects determining the production ratio of isomeric states}

In the measurements reported here possible contaminations are mostly isomeric states, which could not be separated in the preparation Penning trap. Exceptions are the heavy isobars ${ }^{229} \mathrm{Fr}$ and ${ }^{229} \mathrm{Ra}$. The spallation process does not transfer large angular momentum and hence low-spin states are predominantly produced. Fragmentation reactions lead to a production of higher spin states. Nuclides in states with half-lives shorter than few $10 \mathrm{~ms}$ are suppressed due to the slow release time of the reaction products from the ISOLDE target and the time needed for the isobaric separation in the preparation Penning trap (see Fig. 1).

A further selection of an atom in a specific nuclear state can be obtained by resonance laser ionization (RIS) with the ISOLDE laser ion source and a proper choice of laser wavelengths and bandwidths [29]. Due to different hyperfine splittings and isotope shifts in the ground and isomeric state, RIS can enhance the selectivity.

In the case of bismuth investigated in the present work, the lasers were used in the broad-band mode. The production ratios in this experiment can not be deduced from previous measurements with the conventional ion source at ISOLDE. For lead, the applied broad-band mode (laser bandwidth $=10 \mathrm{GHz}$ ) does not result in a selection of isomeric states, since the isomeric shift and hyperfine splitting are smaller or of the same size as the laser bandwidth.

\section{Experimental results}

Table 2 lists all frequency ratios that were determined relative to the cyclotron frequency of ${ }^{133} \mathrm{Cs}$ ions. If possible, the assignment to a particular isomeric state is indicated and will be discussed in Sec. 6. The last column lists all mass excess values deduced from the determined frequency ratios $r$. 
Table 2: Cyclotron frequency ratios $r$ of the ${ }^{133} \mathrm{Cs}^{+}$reference ion with $\nu_{\mathrm{c}, \text { ref }}$ relative to the cyclotron frequency $\nu_{\mathrm{c}}$ of the nuclide under study. The indices $m$ and $x$ behind the element symbol indicate the first excited isomeric state $(\mathrm{m})$ or a possibly unresolved mixture of different states $(\mathrm{x})$. The third column gives the relative total uncertainty $\sigma_{\text {rel }}$. The last column lists the mass excess as derived from $M E=[m$ (in $\mathrm{u})-A]$ (in $\mathrm{keV})$ using the reference mass $m_{\text {ref }}$ of ${ }^{133} \mathrm{Cs}$ ions [49].

\begin{tabular}{|c|c|c|c|}
\hline Nuclide & $\begin{array}{l}\text { Frequency ratio } \\
\qquad r=\frac{\nu_{\mathrm{c}, \mathrm{ref}}}{\nu_{\mathrm{c}}}\end{array}$ & $\sigma_{\text {rel }}$ & $\begin{array}{c}\text { Mass excess } \\
{[\mathrm{keV}]}\end{array}$ \\
\hline${ }^{145} \mathrm{Cs}$ & $1.090516444(91)$ & $8.3 \times 10^{-8}$ & $-60052(11)$ \\
\hline${ }^{147} \mathrm{Cs}$ & $1.10562975(48)$ & $4.3 \times 10^{-7}$ & $-52011(60)$ \\
\hline${ }^{181} \mathrm{Tl}$ & $1.361768475(82)$ & $6.0 \times 10^{-8}$ & $-12802(10)$ \\
\hline${ }^{183} \mathrm{Tl}$ & $1.376786217(81)$ & $5.9 \times 10^{-8}$ & $-16592(10)$ \\
\hline${ }^{186} \mathrm{Tl}^{m}$ & $1.399332234(69)$ & $5.0 \times 10^{-8}$ & $-19874.4(8.6)$ \\
\hline${ }^{187} \mathrm{Tl}^{x}$ & $1.40683800(18)$ & $1.3 \times 10^{-7}$ & $-22154(23)$ \\
\hline${ }^{196} \mathrm{Tl}^{m}$ & $1.474515617(98)$ & $6.7 \times 10^{-8}$ & $-27103(12)$ \\
\hline${ }^{205} \mathrm{Tl}$ & $1.542259739(82)$ & $5.3 \times 10^{-8}$ & $-23818(10)$ \\
\hline${ }^{197} \mathrm{~Pb}^{m}$ & $1.482061386(45)$ & $3.0 \times 10^{-8}$ & $-24429.5(5.5)$ \\
\hline${ }^{208} \mathrm{~Pb}$ & $1.564849038(42)$ & $2.7 \times 10^{-8}$ & $-21742.2(5.2)$ \\
\hline${ }^{190} \mathrm{Bi}^{x}$ & $1.42950438(20)$ & $1.4 \times 10^{-7}$ & $-10535(25)$ \\
\hline${ }^{191} \mathrm{Bi}$ & $1.437006669(64)$ & $4.5 \times 10^{-8}$ & $-13244.9(8.0)$ \\
\hline${ }^{192} \mathrm{Bi}^{m}$ & $1.444529605(72)$ & $5.0 \times 10^{-8}$ & $-13398.5(9.0)$ \\
\hline${ }^{193} \mathrm{Bi}$ & $1.452033781(84)$ & $5.8 \times 10^{-8}$ & $-15875(10)$ \\
\hline${ }^{194} \mathrm{Bi}^{m}$ & $1.45955793(12)$ & $8.0 \times 10^{-8}$ & $-15878(50)^{* *}$ \\
\hline${ }^{195} \mathrm{Bi}$ & $1.467064775(45)$ & $3.1 \times 10^{-8}$ & $-18023.7(5.6)$ \\
\hline${ }^{196} \mathrm{Bi}^{m}$ & $1.47459021(12)$ & $7.9 \times 10^{-8}$ & $-17868(50)^{* *}$ \\
\hline${ }^{197} \mathrm{Bi}^{x}$ & $1.482099666(89)$ & $6.0 \times 10^{-8}$ & $-19706(25) \underline{t \dagger}$ \\
\hline
\end{tabular}

\footnotetext{
** The original error of $14 \mathrm{keV}$ is increased in the AME2003 due to a possible contamination by the $3^{+}$and $10^{-}$states.

†† Value corrected by $-16(22) \mathrm{keV}$ in the AME2003 for possible contamination from ${ }^{197} \mathrm{Bi}^{m}$.
} 
Table 2: (Continued).

\begin{tabular}{lllr}
\hline Nuclide & $\begin{array}{c}\text { Frequency ratio } \\
r=\frac{\nu_{\text {c,ref }}}{\nu_{\mathrm{c}}}\end{array}$ & \multicolumn{1}{c}{$\sigma_{\text {rel }}$} & \multicolumn{1}{c}{$\begin{array}{c}\text { Mass excess } \\
{[\mathrm{keV}]}\end{array}$} \\
\hline${ }^{209} \mathrm{Bi}$ & $1.572401384(35)$ & $2.2 \times 10^{-8}$ & $-18254.9(4.3)$ \\
${ }^{215} \mathrm{Bi}$ & $1.61770721(12)$ & $7.3 \times 10^{-8}$ & $1648(15)$ \\
${ }^{216} \mathrm{Bi}$ & $1.625265520(93)$ & $5.7 \times 10^{-8}$ & $5873(11)$ \\
${ }^{203} \mathrm{Fr}$ & $1.52741073(13)$ & $8.6 \times 10^{-8}$ & $861(16)$ \\
${ }^{205} \mathrm{Fr}$ & $1.542441561(73)$ & $4.7 \times 10^{-8}$ & $-1308.6(9.0)$ \\
${ }^{229} \mathrm{Fr}$ & $1.72332168(30)$ & $1.7 \times 10^{-7}$ & $35816(37)$ \\
${ }^{214} \mathrm{Ra}$ & $1.61017053(17)$ & $1.0 \times 10^{-7}$ & $100(20)$ \\
${ }^{229} \mathrm{Ra}$ & $1.72329550(16)$ & $9.1 \times 10^{-8}$ & $32575(19)$ \\
${ }^{230} \mathrm{Ra}$ & $1.73083537(10)$ & $5.8 \times 10^{-8}$ & $34518(12)$ \\
\hline
\end{tabular}

\section{$5 \quad$ Atomic mass evaluation}

The Atomic Mass Evaluation AME is an adjustment procedure giving mass data for all those nuclides where relevant experimental information, like for example mass values or half-lives, are known. Such an evaluation was performed in December 2002 before these ISOLTRAP data were included. This atomic mass evaluation is referred to in the following as Intermediate Atomic Mass Evaluation 2002 (IAME2002). It is an up-to-date adjustment after the published issue of AME1995 [50] and the NUBASE compilation [47]. The assignment of the observed cyclotron resonances to nuclear states was not straightforward at all since this area of the chart of nuclei is rich in low-lying isomers. Subsequently, the ISOLTRAP results, reported in this publication, were included into the Atomic Mass Evaluation 2003. A detailed description of the adjustment procedure is given in the particular issues of the AME [50,51,52]. Here, only some general features that are relevant for this work are described. As a result of the AME, adjusted mass values are obtained from different types of experimental input data. Even though a possible existence of more than 6000 different combinations of protons and neutrons is predicted, data from only approximately 2830 nuclides are available 53. If direct experimental data are not existing for nuclides near to the region of 


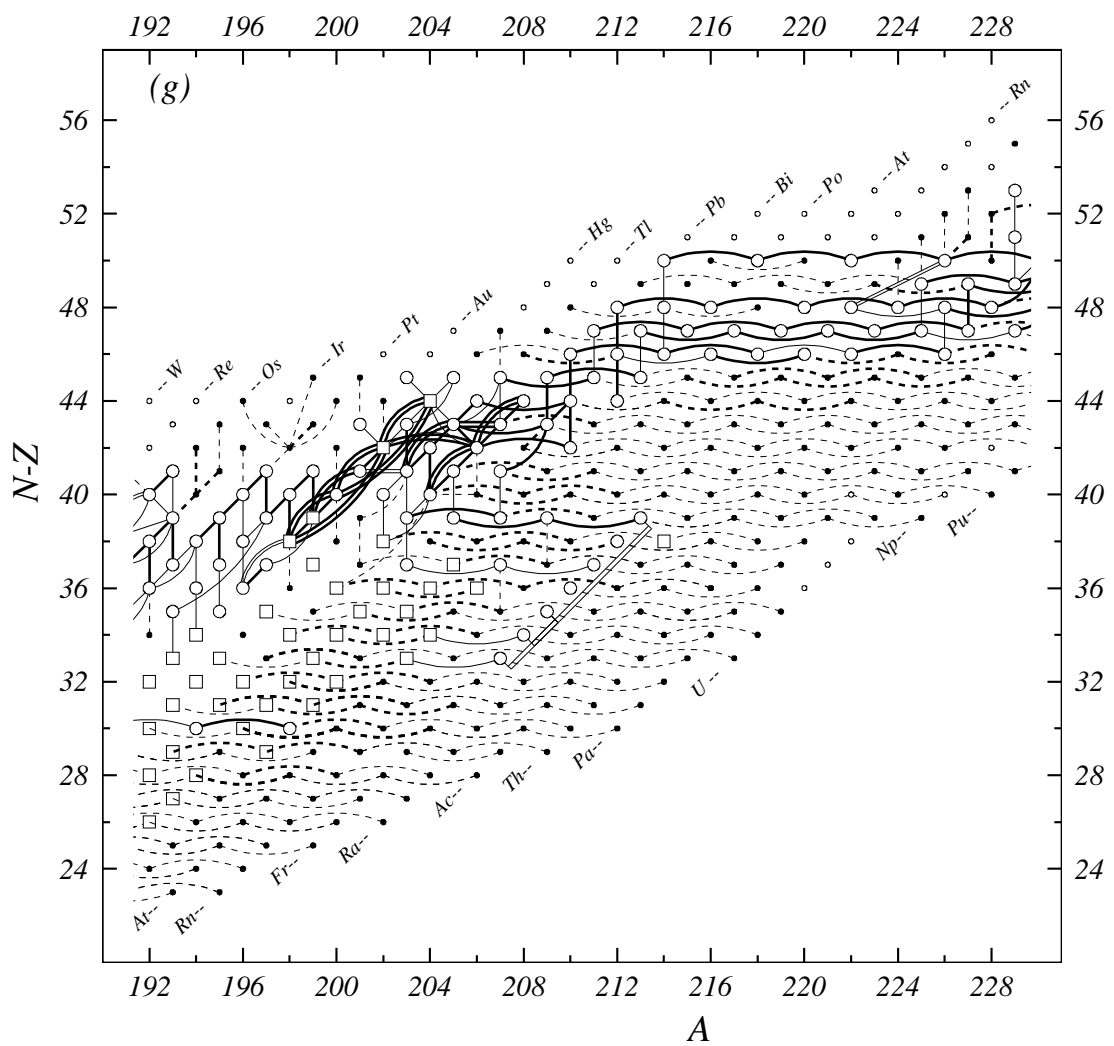

Fig. 4. Connection diagram between nuclides of mass number $A$ and neutron excess $(N-Z)$. The symbols indicate primary nuclides (big symbols, with ' $\square$ ' being an absolute mass-doublet nuclide) and secondary nuclides (small symbols). The latter ones can be distinguished between experimental data (filled) and extrapolated values from systematic trends (open). Primary connections are direct mass spectroscopic data (double line) or reaction data (single line), while thicker lines indicate data with higher precision. All secondary nuclides are connected via dashed lines. This plot is taken from the AME2003 [54.

known masses, mass values are extrapolated from systematic, experimental trends. All experimental results can be represented as a relation between two (and more) nuclides. They span a net of interconnections between nuclides across the whole nuclear chart as it can be seen in the diagram for $A=192$ to $A=229$ (Fig. 4). For the present ISOLTRAP data giving a frequency ratio to a nuclide with precisely known atomic mass value, these connections are linked to the mass of ${ }^{133} \mathrm{Cs}$, which has been determined with a relative uncertainty of only $0.2 \mathrm{ppb}$ [49].

All input data are transformed into a set of linear equations. For the case of experimental frequency ratios this is explained in [55]. Each datum $x_{i}$ with a standard deviation $d x_{i}$ represents a link between two, three, or four unknown masses $m_{\lambda}$. The over-determined set of $X$ equations for $Y$ mass values $(X>Y)$ is represented as a set of linear equations with $Y$ parameters and is finally solved by the least-squares method as described in [53. Most of the ISOLTRAP frequency ratios that are published in the present work were in- 
troduced already into the most recently published atomic mass evaluation, i.e. AME2003 [52].

\section{Assignment of the observed cyclotron resonances to nuclear states}

The assignment of a measured mass value to an isomeric state is performed under the assumption that isomeric states with a given $I^{\Pi}$ and excitation energy might appear as listed in Tab. 1,

\subsection{Nuclides with odd mass number $A$}

In this section those odd-mass nuclides having a long-lived isomeric state are discussed. According to Tab. 1 the ground states of ${ }^{181,183,187} \mathrm{Tl}$ and ${ }^{197} \mathrm{~Pb}$ have always a low spin value, whereas in the bismuth isotopes ${ }^{191,193,195,197} \mathrm{Bi}$ the ground state has a high spin.

The comparison of the results of Tab. 2 with those of the intermediate adjustment (IAME2002) [48, helps to indicate which spin states have most probably been produced during the experiment. Shown in Fig. 5 is such a comparison of the ISOLTRAP masses to the IAME2002 masses with and without the added isomeric energy. The zero line depicts the experimental ISOLTRAP data with their uncertainties. The differences to the literature values are indicated by open symbols, whereas the position of excited nuclear states is taken from the current NUBASE data (Tab. 1) [47,48]. The uncertainty of the excited state is derived by adding the uncertainties of the ground state mass value and the excitation energy quadratically.

${ }^{181} \mathbf{T l}$ and ${ }^{183} \mathrm{Tl}$ : Here, the half-lives of the excited isomeric states $\left(I^{\pi}=9 / 2^{+}\right)$ of $2.7 \mathrm{~ms}$ for ${ }^{181} \mathrm{Tl}$ and $60 \mathrm{~ms}$ for ${ }^{183} \mathrm{Tl}$, are much shorter than the duration of a measurement cycle (approximately $1 \mathrm{~s}$ ). Therefore, the measured cyclotron frequency ratios are clearly assigned to the respective ground states. The measurements of ISOLTRAP agree with the values from the intermediate adjustment IAME2002 (compare Fig. [5), but the uncertainties got significantly improved.

${ }^{187} \mathrm{Tl}$ : The measurement of the nuclide ${ }^{187} \mathrm{Tl}$ has been performed with a mass resolution of $540 \mathrm{keV}$, which is insufficient to resolve both ground and isomeric states, differing by $E=332 \mathrm{keV}$. Hence, an eventually present admixture of either of the states can not be excluded. The determined ISOLTRAP value suggests a predominant production of the isomer. 


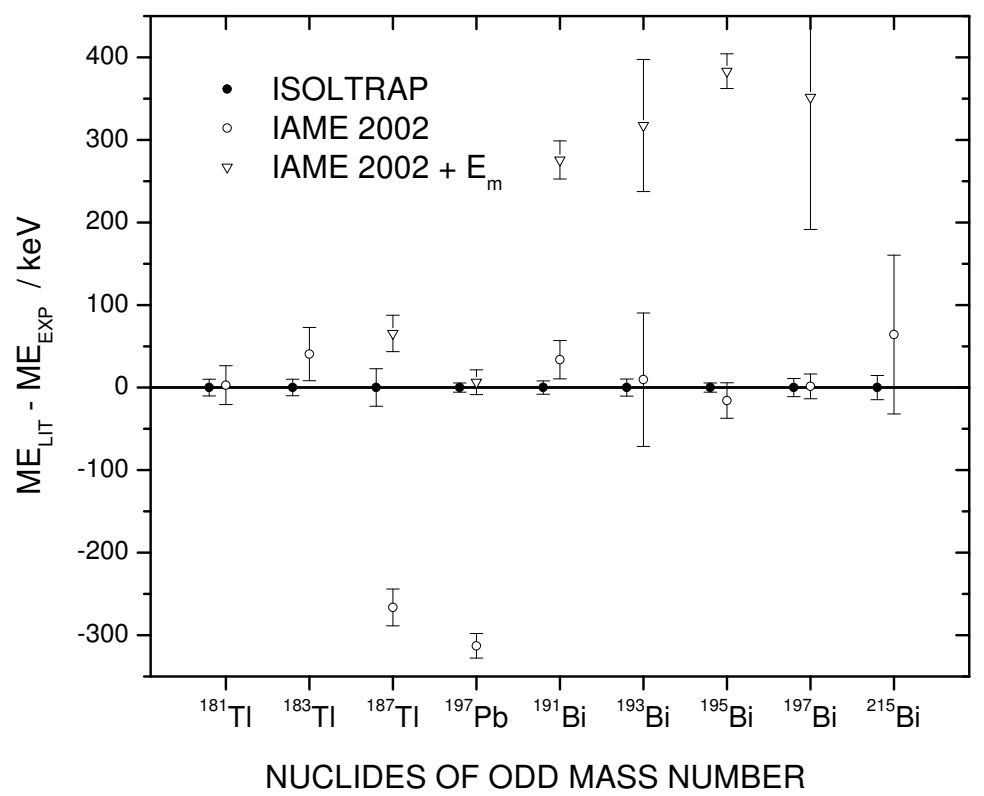

Fig. 5. Comparison of ISOLTRAP mass values for odd-even and even-odd nuclides to literature values. The zero line represents the experimental ISOLTRAP data with their uncertainties. Open symbols indicate the results of an intermediate atomic mass evaluation performed end of 2002 (IAME2002). IAME2002 includes all literature values available at that time but not the ISOLTRAP data. The excitation energies $E_{\mathrm{m}}$ are taken from NUBASE [47/48].

${ }^{197} \mathrm{~Pb}^{m}$ : The mass values of both ground and isomeric states in ${ }^{197} \mathrm{~Pb}$ are listed in IAME2002 with uncertainties of only $15 \mathrm{keV}$. The value resulting from the ISOLTRAP measurement with a final uncertainty of $5.5 \mathrm{keV}$ agrees within the error bars to the one of the excited $I^{\Pi}=13 / 2^{+}$state $(E=319.3 \mathrm{keV})$ and hence is assigned to it.

${ }^{191,193,195,197} \mathbf{B i},{ }^{215} \mathbf{B i}$ : A series of bismuth isotopes with odd mass numbers $A=191$ to 197 and $A=215$ has been studied. With the exception of the case of ${ }^{197} \mathrm{Bi}$ the mass resolution $\Delta m$ (see Tab. 11) is at least a factor of two smaller than the mass difference between both states. Therefore, a contamination of the respective second state is definitely excluded in the count-rate-class analysis. The remaining question which of the two states has been measured cannot be solved easily. Yield measurements for lead and bismuth isotopes at ISOLDE reveal that states having higher spin values are predominantly produced [30]. These are for $191 \leq A \leq 197$ the $I^{\Pi}=9 / 2^{-}$ground states of bismuth. Indeed, the comparison of the ISOLTRAP results shows a good agreement with the values of the intermediate evaluation IAME2002 for the ground states of ${ }^{191,193,195,197} \mathrm{Bi}$. The measurements of ${ }^{197} \mathrm{Bi}$ were performed with a resolution 
of $600 \mathrm{keV}$ which is not sufficient to resolve ground and isomeric state, differing by $350(160) \mathrm{keV}$. Measurements of the neighboring odd- $A$ isotopes ${ }^{193} \mathrm{Bi}$ and ${ }^{195} \mathrm{Bi}$ with approximately $115 \mathrm{keV}$ resolution did not show any hint of a contamination by an excited state. A systematic comparison of individual measurements with low $(580 \mathrm{keV})$ and high $(115 \mathrm{keV})$ resolution was used in order to quantify a correction by a possible contamination by the excited ${ }^{197} \mathrm{Bi}^{m}$ state. This correction of $-16(22) \mathrm{keV}$ increases the uncertainty of the ISOLTRAP value in the atomic mass table AME2003 from $11 \mathrm{keV}$ to $25 \mathrm{keV}$. Considering the isomeric states of all odd-mass nuclides studied here a consistent picture is observed. Except for cases where the presence of a state can be excluded right away due to its short half-life, higher spin states are predominantly produced. In ${ }^{187} \mathrm{Tl}$ and ${ }^{197} \mathrm{~Pb}$ these are the excited states with spins of $9 / 2^{-}$and $13 / 2^{+}$. In bismuth $(A \leq 197)$ the ground states have a higher spin value of $9 / 2^{-}$. For the nuclide ${ }^{215} \mathrm{Bi}$ with an excited state of $>1 \mathrm{MeV}$ energy and a spin $25 / 2^{-}$, the $9 / 2^{-}$ground state mass value has been determined.

\subsection{Nuclides with even mass number $A$}

In order to facilitate a spin-to-state assignment, the present ISOLTRAP data are plotted in Fig. [6] and compared relative to ground state mass values and excitation energies from the intermediate evaluation IAME2002. These nuclides have sometimes two excited, long-lived states (see also Tab. 1). Excitation energies of states with half-lives longer than 500 ns are shown.

Apart from the 500 ns-state of ${ }^{190} \mathrm{Bi}$ all nuclides have half-lives that are sufficiently long $(\geq 50 \mathrm{~ms}$ ) in order to be studied with the Penning trap mass spectrometer ISOLTRAP. Due to the lack of any quantitative information on production ratios, a conclusion can be only based on a systematic comparison within the current data. In analogy to the nuclides of odd mass number, it is expected that lower spin states will be produced with lower yields.

${ }^{186} \mathrm{Tl}^{m}$ : An unambiguous assignment of the ISOLTRAP mass value to one of the long-lived states in ${ }^{186} \mathrm{Tl}$ is not straightforward. This is also reflected by the different spin assignments given in NUBASE (Tab. 1) and in the Evaluated Nuclear Structure Data Files (ENSDF). Mass values of both excited states are given with uncertainties of $50 \mathrm{keV}$. The energy difference between ${ }^{186} \mathrm{Tl}^{m}$ and ${ }^{186} \mathrm{Tl}^{n}$ is $373.9(5) \mathrm{keV}$ [16]. The attempt to employ a very long RF-excitation of $9 \mathrm{~s}$, corresponding to a mass resolution of $\Delta m=33 \mathrm{keV}$, failed. For excitation times beyond $3 \mathrm{~s}$, a decay of the stored ions has been observed (see half-lives in Tab. 1).

Figure 7 shows the results of the four cyclotron frequency measurements. With a mass resolution of $210 \mathrm{keV}$ (measurement \# 2 - 4) a mixture between ${ }^{186} \mathrm{Tl}^{m}$ and ${ }^{186} \mathrm{Tl}^{n}$ is resolved and the presence of either of the states should have been 


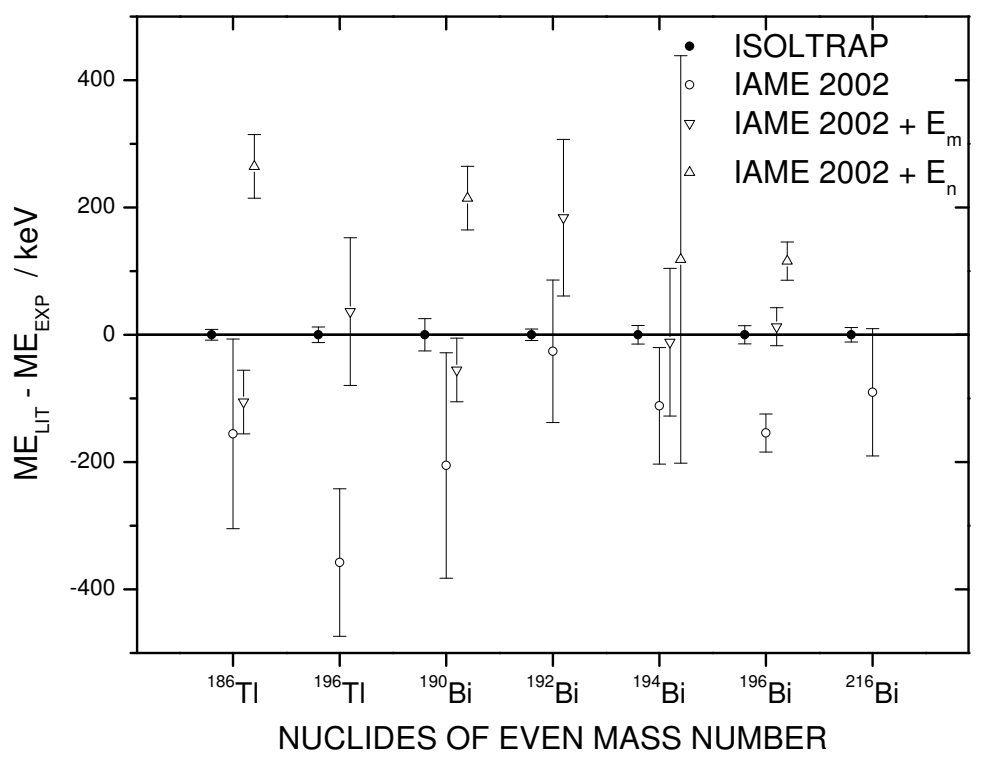

Fig. 6. Comparison of even- $A$ mass values from ISOLTRAP to an intermediate atomic mass evaluation (IAME2002). The zero line represents the experimental data points obtained within this work with their uncertainties. Excited, long-lived states as they are listed in NUBASE [47/48] are shown by open triangles. Those isomeric states with the higher excitation energy $E_{\mathrm{n}}$ are depicted by a triangle pointing upwards.

detected. Measurement \# 2 and \# 4 yield count-rate-class corrected frequencies. The slope $z$ in $[\mathrm{mHz} / \mathrm{ion}]$ of the linear extrapolation of the cyclotron frequency of ${ }^{186} \mathrm{Tl}^{+}$is indicated for each measurement. The third data point (\# 3), which has been obtained after an additional waiting time of $10 \mathrm{~s}$ in the upper precision trap, does not show a significant count rate effect, since this measurement allowed for a decay of the $T_{1 / 2}=2.9$ s-state. To avoid any undetected contamination from the low-resolution measurement \# 1, it was not considered in the final weighted average of the frequency ratio. After a comparison to the spin systematics, as given in Tab. 1, and the agreement of the ISOLTRAP value with the intermediate evaluation IAME2002 this result is assigned to the ${ }^{186} \mathrm{Tl}^{m}$ state. It is not excluded that a small contamination by the ground state $(\Delta E=40 \mathrm{keV})$ might be present.

${ }^{196} \mathrm{Tl}^{m}$ : The mass resolution, being $120 \mathrm{keV}$ during this measurement, was sufficiently high to separate both states of ${ }^{196} \mathrm{Tl}$. The resulting ISOLTRAP value agrees with the mass value of the excited, medium spin state ${ }^{196} \mathrm{Tl}^{m}$ $\left(T_{1 / 2}=1.41 \mathrm{~h}, I^{\pi}=\left(7^{+}\right)\right)$though the uncertainties of the IAME2002 values are rather large.

190,194,196 Bi: These nuclides have three long-living states similar to almost all even- $A$ bismuth isotopes up to ${ }^{212} \mathrm{Bi}$. The excitation energies of about $150 \mathrm{keV}$ 


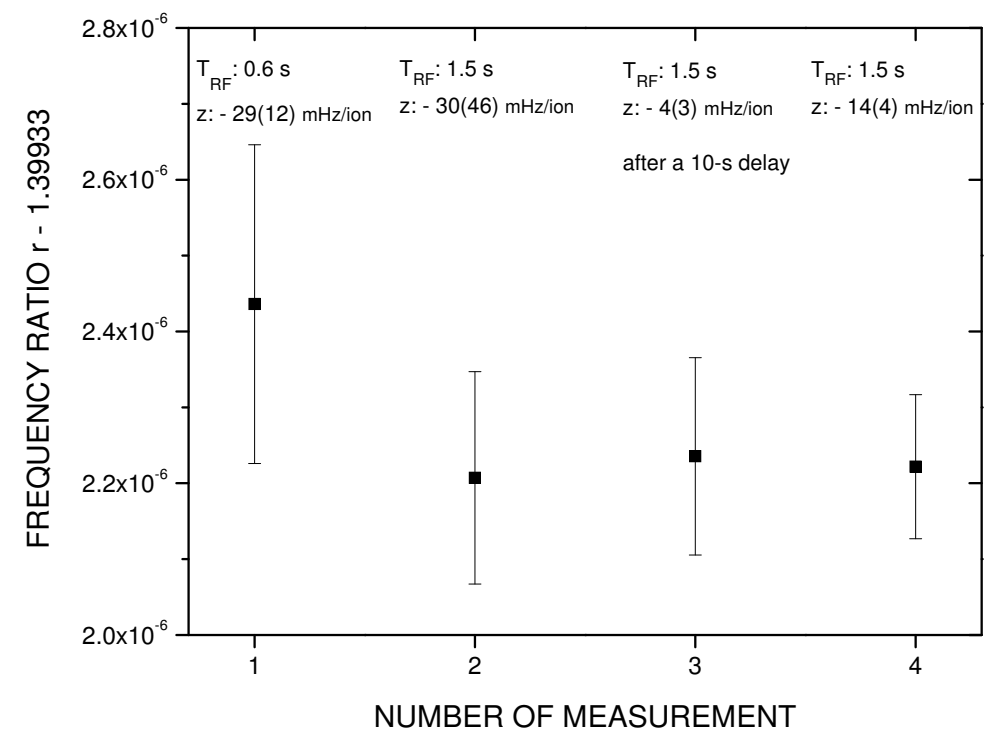

Fig. 7. Four cyclotron frequency measurements for ${ }^{186} \mathrm{Tl}$ with two different excitation times $T_{\mathrm{RF}}$ as indicated. $T_{\mathrm{RF}}=0.6 \mathrm{~s}$ corresponds to a mass resolution of $\Delta m=530 \mathrm{keV}$ and $T_{\mathrm{RF}}=1.5 \mathrm{~s}$ to $\Delta m=210 \mathrm{keV}$. The result of the count-rateclass analysis of the cyclotron resonance of ${ }^{186} \mathrm{Tl}$ is given for each data point. In the measurement \#3 an additional waiting time in the precision trap of $10 \mathrm{~s}$ has been employed prior to the frequency determination.

are all known with uncertainties of around $70 \mathrm{keV}$, except for ${ }^{196} \mathrm{Bi}$ (Fig. 6). The resolving power employed during those cyclotron frequency measurements of approximately $3 \times 10^{5}$ corresponds to a mass resolution between 560 and $590 \mathrm{keV}$. In order to obtain a mass resolution of $50 \mathrm{keV}$ an excitation time of $7 \mathrm{~s}$ is required.

The mass value of ISOLTRAP for ${ }^{196} \mathrm{Bi}$ agrees well to the one of the $0.6 \mathrm{~s}$ state $\left({ }^{196} \mathrm{Bi}^{m}\right)$. However, a contamination cannot be excluded. Accounting for a possible admixture of either the $3^{+}$or the $10^{-}$state the original uncertainty of $14 \mathrm{keV}$ had been increased to $50 \mathrm{keV}$ in the AME2003.

Due to the good agreement for ${ }^{196} \mathrm{Bi}$, it is concluded that the medium spin state has been predominantly produced for ${ }^{194} \mathrm{Bi}$ as well. Since the possible presence of both isomeric states, $3^{+}$and $10^{-}$, whereas each of them is about $100 \mathrm{keV}$ different in energy, cannot be excluded with a resolution of $580 \mathrm{keV}$, the original error of $14 \mathrm{keV}$ is increased in the Atomic Mass Evaluation 2003 to $50 \mathrm{keV}$.

In ${ }^{190} \mathrm{Bi}$ the medium spin state exhibits a half-life in the nanoseconds regime. The measured ISOLTRAP value with $25 \mathrm{keV}$ uncertainty agrees within $1 \sigma$ to the value of the $6.2 \mathrm{~s}$-state $\left({ }^{190} \mathrm{Bi}\right)$. Due to the minor resolution during the measurement, a mixture of both states ${ }^{190} \mathrm{Bi}$ and ${ }^{190} \mathrm{Bi}^{m}$ differing by $E=150(190) \mathrm{keV}$ might be present. 
${ }^{192} \mathrm{Bi}$ : In contrast to other even- $A$ bismuth isotopes from ${ }^{190} \mathrm{Bi}$ to ${ }^{210} \mathrm{Bi}$, no medium nuclear spin of, for example, $I=6$ or $I=7$ has been observed. However, the general trend in this experiment points to a favored production of medium spin states, $I=9 / 2$ or 7 . The question on the production ratio between the $3^{+}$ground state and the excited $10^{-}$state remains unsolved. From the general trend throughout all these measurements it is observed, that it is the higher spin state that is produced with a higher weight and measured in the trap. Consequently the mass value has been tentatively assigned to ${ }^{192} \mathrm{Bi}^{m}$ in the Atomic Mass Evaluation 2003. In the mass measurement of this nuclide two observations are made: First, the counting class analysis of two individual measurements with a resolution of $\Delta m=110 \mathrm{keV}$ indicate the presence of a contamination and give a shifted, extrapolated value. Secondly, the characteristic pattern for an in-trap decay with short half-life is observed in the time-of-flight spectrum although the half-lives of both known states are more than ten times longer than the excitation time of $T_{\mathrm{RF}}=3 \mathrm{~s}$. This might indicate a third, so far unknown, isomeric state with shorter half-life or any other contamination.

\subsection{Isobaric nuclides ${ }^{229} \mathbf{F r}$ and ${ }^{229} \mathbf{R a}$}

The nuclides ${ }^{229} \mathrm{Fr}$ and ${ }^{229} \mathrm{Ra}$ were stored simultaneously in the precision trap since the applied mass resolving power $R \approx 4 \times 10^{4}$ in the preparation Penning trap was not sufficient to separate the $A=229$-isobars. Figure 8 shows the cyclotron resonance curves of the isobars, which differ in mass by $3.2 \mathrm{MeV}$. The

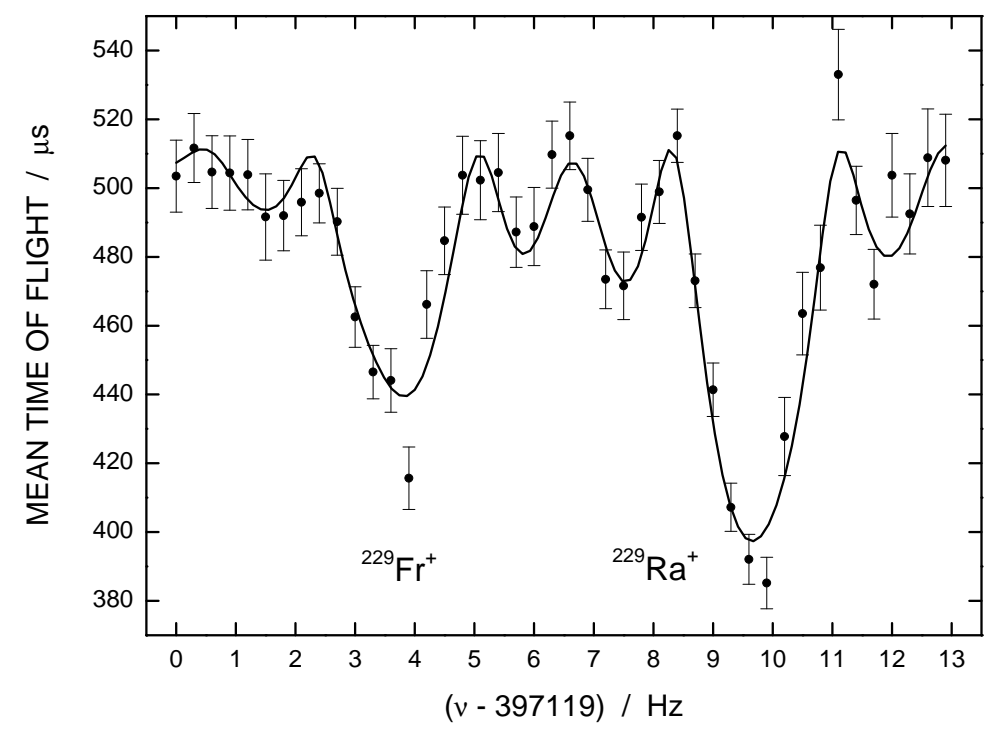

Fig. 8. Cyclotron resonance curves of the isobars ${ }^{229} \mathrm{Fr}$ (left) and ${ }^{229} \mathrm{Ra}$ (right). Both isobars were simultaneously delivered from the preparation trap to the precision trap.

center frequencies including the first sidebands are clearly separated with a 


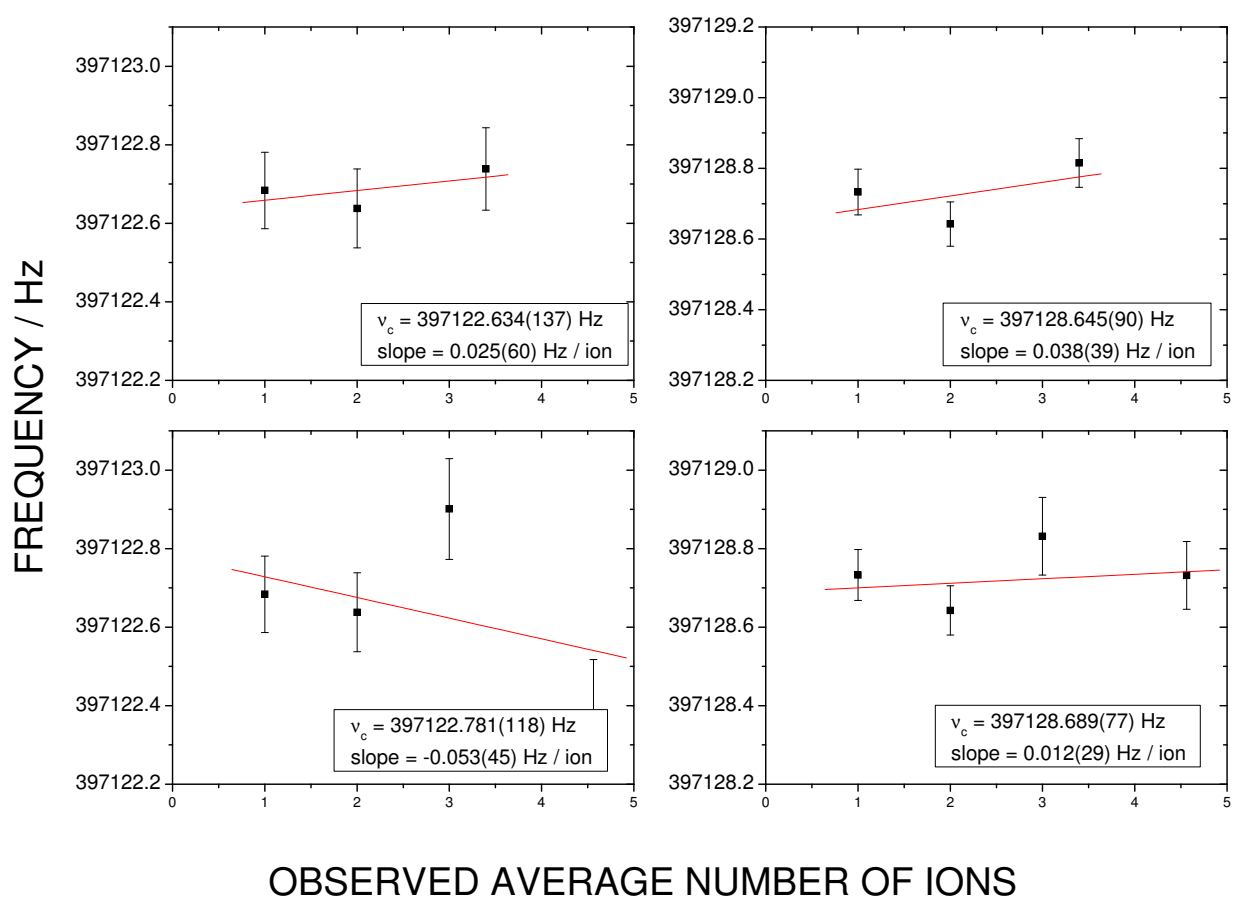

Fig. 9. Count-rate-class analysis for isobaric ions ${ }^{229} \mathrm{Fr}$ (left) and ${ }^{229} \mathrm{Ra}$ (right) of the signal shown in Fig. 8, All registered ions were subdivided into three (upper diagrams) as well as into four (lower diagrams) count rate classes. The data points are fitted by linear regression. The resulting slopes and center frequencies of the cyclotron resonances are given in the inset. None of the individual measurements shows a significant variation of the cyclotron frequency on the number of detected ions.

resolving power of $R \approx 2.4 \times 10^{5}$, using an excitation time of $T_{\mathrm{RF}}=600 \mathrm{~ms}$. A superposition of two individual cyclotron resonance curves, weighed by their relative abundance, was fitted to the data points. Assuming a detection efficiency of the microchannel plate of $30 \%$, the average number of detected ions of 2.5 corresponds to about eight ions that were simultaneously stored. Therefore, the possibility of a frequency shift dependent on the number of ions (see Sec. 3.2) was investigated in a detailed count-rate-class analysis.

The result of such an analysis is plotted in Fig. 9 for the cyclotron frequencies of ${ }^{229} \mathrm{Fr}$ (left) and ${ }^{229} \mathrm{Ra}$ (right). Here, a total number of 2340 registered ions was subdivided into three (upper diagrams) as well as into four (lower diagrams) count-rate classes. Different subdivisions were made in order to exclude any possible count-rate dependance produced within the statistics. In both cases no significant count-rate dependance is observed. 


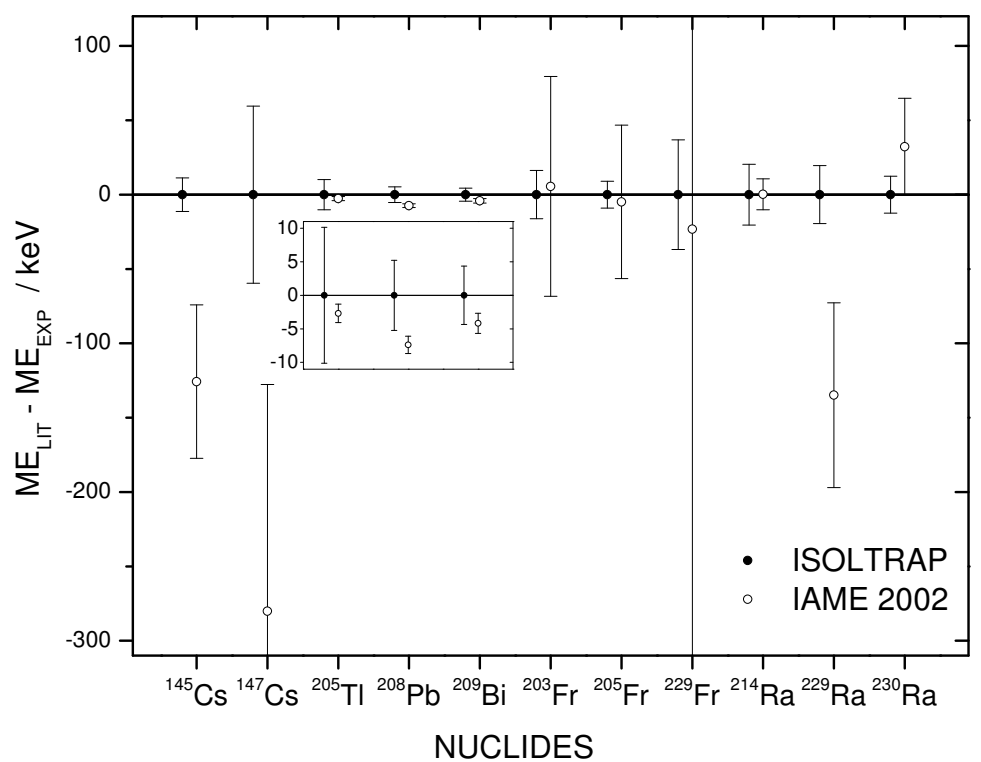

Fig. 10. Comparison of ISOLTRAP mass values to the intermediate adjustment of the Atomic Mass Evaluation IAME2002. The line at zero represents the experimental ISOLTRAP values with their uncertainties. The inset shows an enlarged scale for the stable nuclides.

\subsection{Other nuclides}

Figure 10 shows the mass excess values of other nuclides studied in this work, including the stable isotopes ${ }^{205} \mathrm{Tl},{ }^{208} \mathrm{~Pb}$, and ${ }^{209} \mathrm{Bi}$. The masses of these stable isotopes, which are known with high accuracy, agree within one standard deviation with IAME2002. The largest discrepancies are observed for ${ }^{145} \mathrm{Cs}$ $(-2.4 \sigma),{ }^{147} \operatorname{Cs}(-1.7 \sigma)$, and ${ }^{229} \operatorname{Ra}(-2.0 \sigma)$. 


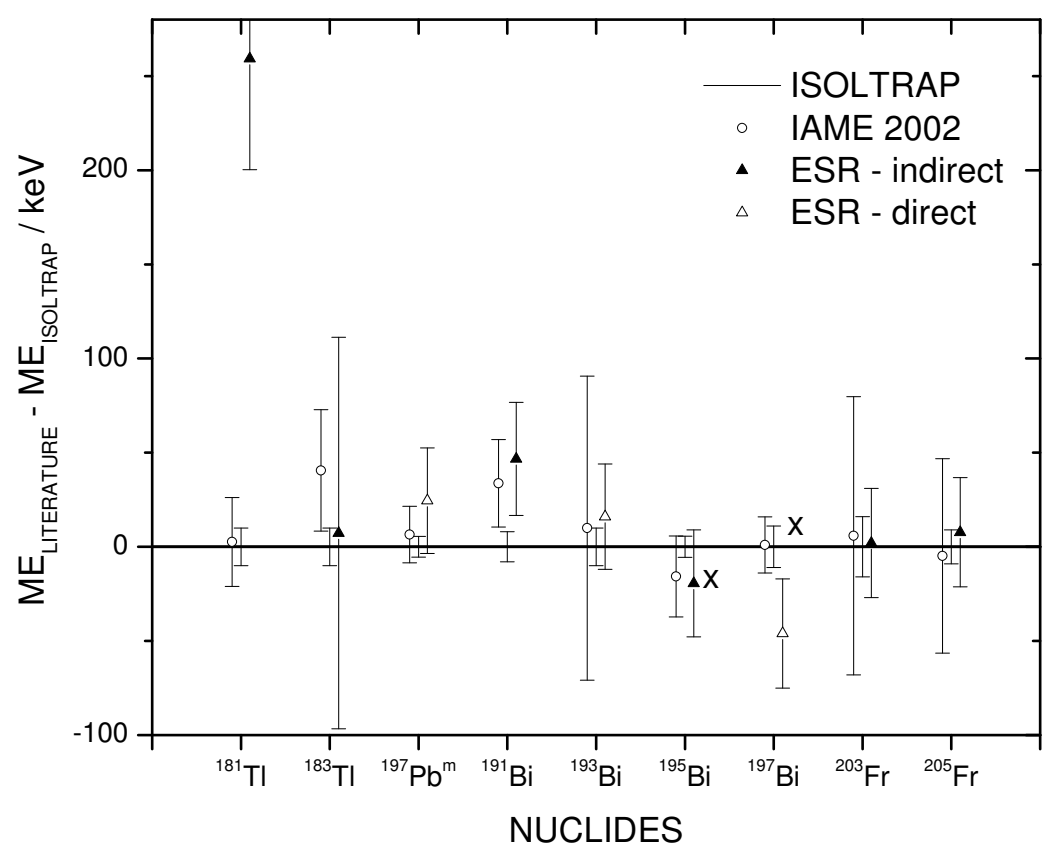

Fig. 11. Comparison of ISOLTRAP, ESR data, and the values of the intermediate adjustment IAME2002 [48]. The ESR values are distinguished between direct measurements (open triangles) and indirect (closed triangles) data which are linked to direct ESR measurements by $Q$ values from literature. All directly determined ESR values are taken from [21, whereas the indirectly determined ones are from [20]. For some of the latter cases a direct value of a mixture of isomeric states is given in [21. The symbol ' $x$ ' denotes those nuclides where an isomeric mixture has not been resolved.

\subsection{Comparison to other experimental data}

Some of the mass values which are reported in this work have also been determined by Schottky mass spectrometry in the Experimental Storage Ring ESR at GSI [20,21]. Figure 11] compares the results of both experiments and the values of the IAME2002. A larger set of data is obtained by including also the indirectly determined results at ESR using $Q$ values from literature [20]. In most of the cases the data from both measurement techniques agree within their uncertainties. An exception is the nuclide ${ }^{181} \mathrm{Tl}$, where both values differ by more than $4.3 \sigma$. Since the ESR value has been indirectly determined, this discrepancy could be attributed as well to the $Q_{\alpha}$ value. 


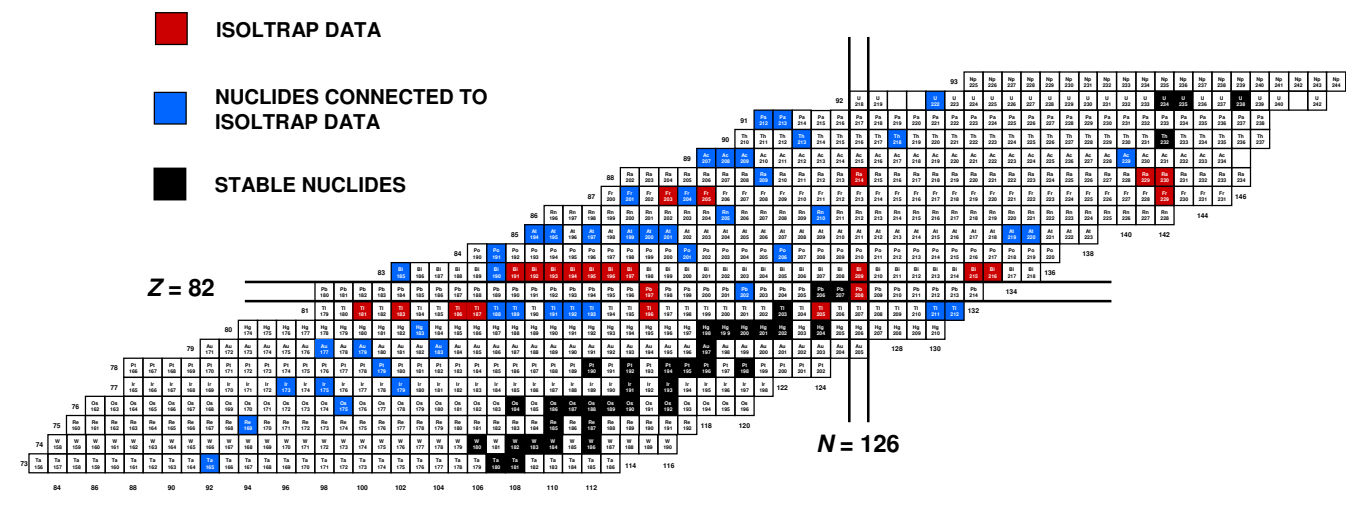

Fig. 12. Region of the nuclear chart around $Z=82$ and $N=126$, indicating the nuclides measured by ISOLTRAP (in red). All other nuclides which are connected to ISOLTRAP data are shown (in blue). Color figure online.

\section{Results}

Table 3 compiles the mass excesses of those radionuclides discussed here and compares the data with values given in NUBASE 1997 [47] and [48]. In addition, the results of the atomic mass evaluation published in 2003 (AME2003) are given, which include the ISOLTRAP results. Due to the manifold interconnections, the present ISOLTRAP data improve not only the directly measured nuclides, but also a large region from $Z=73$ to $Z=92$ interconnected via $\alpha$ chains in particular. Fig. 12 shows the respective section of the nuclear chart from tantalum to neptunium isotopes, indicating all related nuclides in this region.

For primary nuclides, which directly contribute to the adjustment procedure, the total influence of each input value in the AME is given in Tab. 3 by the significance in per cent. It is the sum of all influences on individual mass values $m_{\lambda}$. The quantity ' $\mathrm{v} / \mathrm{s}$ ' denotes the difference between both values, divided by the uncertainty of the ISOLTRAP value. The last column marks those data which were not used in the adjustment procedure. This is the case, if, for example, a contamination of the ISOLTRAP measurement by a further isomeric state could not be excluded, or if, for the stable nuclides, another high-accuracy mass determination already exists, which is at least three times more precise than the ISOLTRAP value.

In addition, the mass values of around 100 other nuclides changed after the atomic mass evaluation with the ISOLTRAP data reported here. The largest modifications in the adjusted mass values is observed for ${ }^{145} \mathrm{Ba}(7 \sigma)$. 
Table 3: ISOLTRAP mass data compared to the data given in NUBASE [47] and [48, and the Atomic Mass Evaluation AME2003 [52]. Here, mass excess values are rounded as described at p. 344 in 52. The significance of the ISOLTRAP measurement is listed in column 'Sign.'. Column 'v/s' denotes the deviation between the mass value as determined by ISOLTRAP and the AME2003 divided by the uncertainty in the input value. The following abbreviations are used in the column 'Remark': 'U' denotes nuclides, that have not been used in the adjustment procedure due to their low impact. ' $F$ ' indicates nuclides with a possible presence of a contamination which, for this reason, are excluded from the adjustment. 'E': The ISOLTRAP value determines the excited ${ }^{196} \mathrm{Tl}^{m}$ state with an excitation energy of $E=394.2(5) \mathrm{keV}$, whereas the mass for the ground state has been implemented in the AME.

\begin{tabular}{|c|c|c|c|c|c|c|}
\hline \multirow[t]{2}{*}{ Nuclide } & \multicolumn{3}{|c|}{ Mass excess $[\mathrm{keV}]$} & \multirow{2}{*}{$\begin{array}{c}\text { Sign. } \\
\%\end{array}$} & \multirow[t]{2}{*}{$\mathrm{v} / \mathrm{s}$} & \multirow[t]{2}{*}{ Remark } \\
\hline & ISOLTRAP & NUBASE1997 and [48] & AME2003 & & & \\
\hline${ }^{145} \mathrm{Cs}$ & $-60052(11)$ & $-60180(50)$ & $-60057(11)$ & 94 & -0.4 & \\
\hline${ }^{147} \mathrm{Cs}$ & $-52011(60)$ & $-52290(150)$ & $-52020(50)$ & 79 & -0.1 & \\
\hline${ }^{181} \mathrm{Tl}$ & $-12802(10)$ & $-12490 \#(300 \#)$ & $-12801(9)$ & 92 & -0.2 & \\
\hline${ }^{183} \mathrm{Tl}$ & $-16592(10)$ & $-16290 \#(190 \#)$ & $-16587(10)$ & 91 & 0.4 & \\
\hline${ }^{186} \mathrm{Tl}^{m}$ & $-19874.4(8.6)$ & $-19980(50)$ & $-19874(9)$ & 100 & & \\
\hline${ }^{187} \mathrm{Tl}^{m}$ & $-22154(23)$ & $-22085(22)$ & $-22109(8)$ & & 2 & $\mathrm{~F}$ \\
\hline${ }^{196} \mathrm{Tl}^{(m)}$ & $-27103(12)$ & $-27470 \#(100 \#)$ & $-27497(12)$ & 100 & & $\mathrm{E}$ \\
\hline${ }^{205} \mathrm{Tl}$ & $-23818(10)$ & $-23820.8(1.4)$ & $-23820.6(1.3)$ & & -0.3 & $\mathrm{U}$ \\
\hline${ }^{197} \mathrm{~Pb}^{m}$ & $-24429.5(5.5)$ & $-24423(15)$ & $-24429(6)$ & 100 & & \\
\hline${ }^{208} \mathrm{~Pb}$ & $-21742.2(5.2)$ & $-21749.6(1.3)$ & $-21748.5(1.2)$ & & -1.2 & $\mathrm{U}$ \\
\hline${ }^{190} \mathrm{Bi}^{m}$ & $-10535(25)$ & $-10590(50)$ & $-10483(10)$ & & 2.1 & $\mathrm{~F}$ \\
\hline${ }^{191} \mathrm{Bi}$ & $-13244.9(8.0)$ & $-13211(23)$ & $-13240(7)$ & 86 & 0.6 & \\
\hline${ }^{192} \mathrm{Bi}^{m}$ & $-13398.5(9.0)$ & $-13220 \#(120 \#)$ & $-13399(9)$ & 100 & & 於 \\
\hline${ }^{193} \mathrm{Bi}$ & $-15875(10)$ & $-15870(80)$ & $-15873(10)$ & 100 & 0.2 & \\
\hline
\end{tabular}

¥¥ Tentative assignment 
Table 3: (Continued).

\begin{tabular}{|c|c|c|c|c|c|c|}
\hline \multirow[t]{2}{*}{ Nuclide } & \multicolumn{3}{|c|}{ Mass excess $[\mathrm{keV}]$} & \multirow{2}{*}{$\begin{array}{c}\text { Sign. } \\
\%\end{array}$} & \multirow[t]{2}{*}{$\mathrm{v} / \mathrm{s}$} & \multirow[t]{2}{*}{ Remark } \\
\hline & ISOLTRAP & NUBASE1997 and [48] & AME2003 & & & \\
\hline${ }^{194} \mathrm{Bi}^{m}$ & $-15878(50)$ & $-15970 \#(440 \#)$ & $-15880(50)$ & 100 & & \\
\hline${ }^{195} \mathrm{Bi}$ & $-18023.7(5.6)$ & $-18039(21)$ & $-18024(6)$ & 100 & & \\
\hline${ }^{196} \mathrm{Bi}^{m}$ & $-17868(50)$ & $-17856(30)$ & $-17842(25)$ & 100 & 0.5 & \\
\hline${ }^{197} \mathrm{Bi}$ & $-19706(25)$ & $-19689(15)$ & $-19688(8)$ & 100 & 0.8 & \\
\hline${ }^{209} \mathrm{Bi}$ & $-18254.9(4.3)$ & $-18259.1(1.5)$ & $-18258.5(1.4)$ & & -0.8 & $\mathrm{U}$ \\
\hline${ }^{215} \mathrm{Bi}$ & $1648(15)$ & $1710(100)$ & $1649(15)$ & 100 & & \\
\hline${ }^{216} \mathrm{Bi}$ & $5873(11)$ & $5780 \#(100 \#)$ & $5874(11)$ & 100 & & \\
\hline${ }^{203} \mathrm{Fr}$ & $861(16)$ & $870(70)$ & $861(16)$ & 100 & & \\
\hline${ }^{205} \mathrm{Fr}$ & $-1308.6(9.0)$ & $-1310(50)$ & $-1310(8)$ & 100 & -0.1 & \\
\hline${ }^{229} \mathrm{Fr}$ & $35816(37)$ & $35790 \#(360 \#)$ & $35820(40)$ & 100 & & \\
\hline${ }^{214} \mathrm{Ra}$ & $100(20)$ & $100(10)$ & 101(9) & 100 & 0 & \\
\hline${ }^{229} \mathrm{Ra}$ & $32575(19)$ & $32440(60)$ & $32563(19)$ & 91 & -0.6 & \\
\hline${ }^{230} \mathrm{Ra}$ & $34518(12)$ & $34550(30)$ & $34518(12)$ & 100 & & \\
\hline
\end{tabular}

\section{Discussion}

\subsection{Irregularities of the mass surface}

The mass values and the corresponding nuclear binding energies represent the underlying forces acting in a nucleus. In analogy to the ionization potential of atoms, plots of separation energies for the removal of two protons,

$$
S_{2 \mathrm{p}}=B E(Z, N)-B E(Z-2, N)
$$

or two neutrons,

$$
S_{2 \mathrm{n}}=B E(Z, N)-B E(Z, N-2)
$$


reveal the details of nuclear shell structure. In this representation, odd-even effects caused by the usually much larger binding energy for even proton or neutron numbers are removed.

Figures 13 and 14 show the behavior of separation energies for protons and neutrons versus the respective nucleon number. The results from AME1995 and AME2003 are compared. A global trend, a continuous decrease in the separation energies, is observed. Discontinuities from this general trend appear at shell closures or in regions of strong deformations. The $N=126$ shell closure is clearly visible in Fig. 13. The much less pronounced strength of the proton shell closure $Z=82$ is larger at the magic neutron number $N=$ 126 than at the half-filled shell $N=104$ (Fig. 14). The main differences between the appearance of the mass surface in 1995 and 2003 are substantially reduced uncertainties, due to improved as well as numerous new experimental input data. These are ISOLTRAP measurements in the isotopic chains of mercury, platinum, lead, polonium, radon, and radium [12]13,56] as well as a large number of new results obtained at the ESR by the Schottky technique [19,20,21. In particular the small uncertainty $(\delta m \approx 25 \mathrm{keV})$ of data from ISOLTRAP along the extended isotopic chain from ${ }^{179} \mathrm{Hg}$ to ${ }^{195} \mathrm{Hg}$ enables the observation of finer nuclear structure effects and to study, for example, nuclear shape coexistence and the influence of low-excitation intruder states [13,14,57]. Note the pronounced irregularities in the thallium isotopic chain at neutron numbers $N=99,105,107$, and 114 .

The two-proton separation energies are much more regular and smooth in this region of the nuclear chart than those for two neutrons. However, a peculiar irregularity in the two-proton separation energies appears for the isotopes of thallium $(Z=81)$ with even neutron numbers $N$ from 98 to 108: Some separation energies are nearly equal or even higher than those of the isotopes with one additional neutron. Such an effect is very uncommon in the chart of nuclei. 

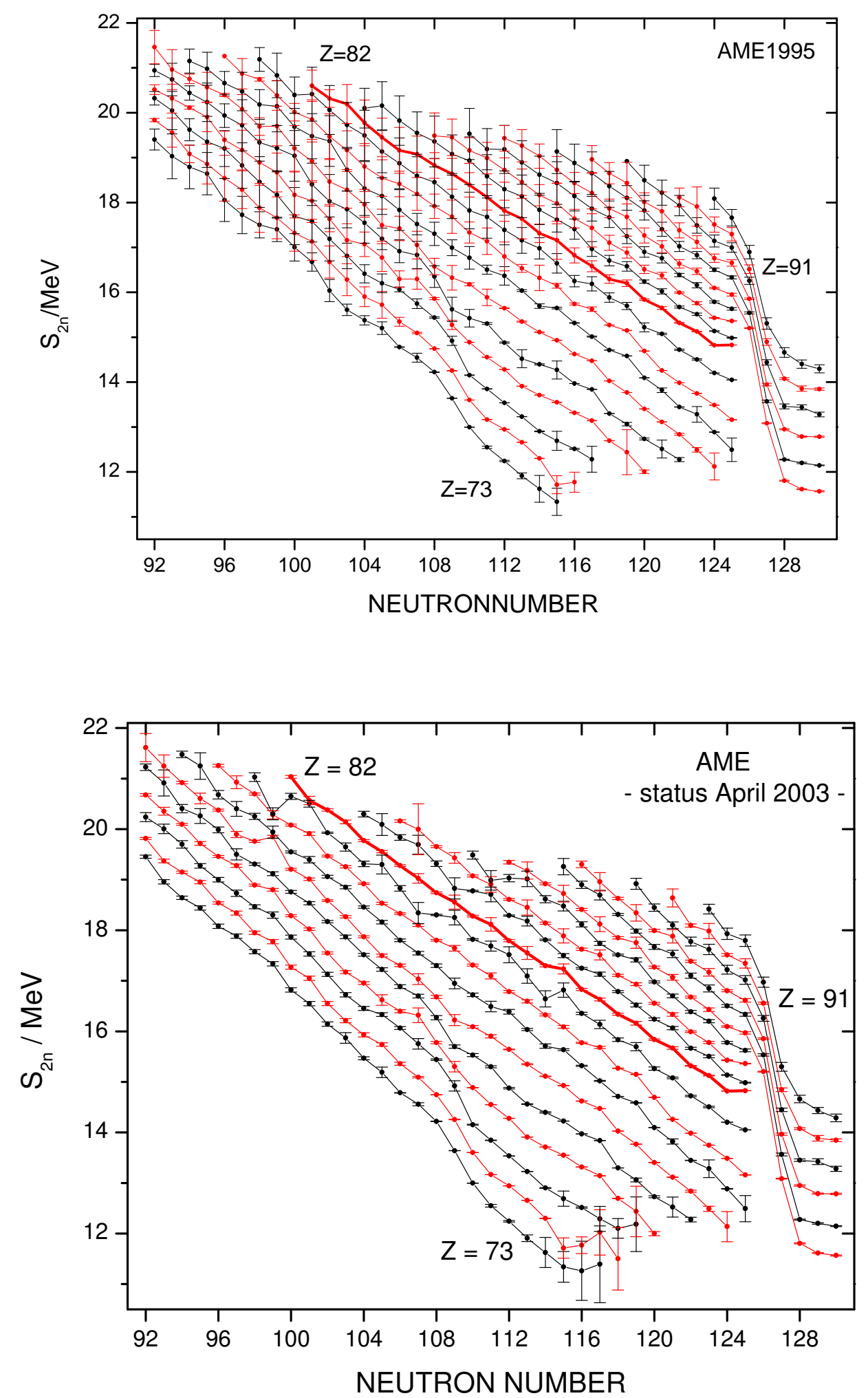

Fig. 13. Comparison of two-neutron separation energies $S_{2 n}$ as obtained in AME1995 [50] and 2003. Color figure online. 

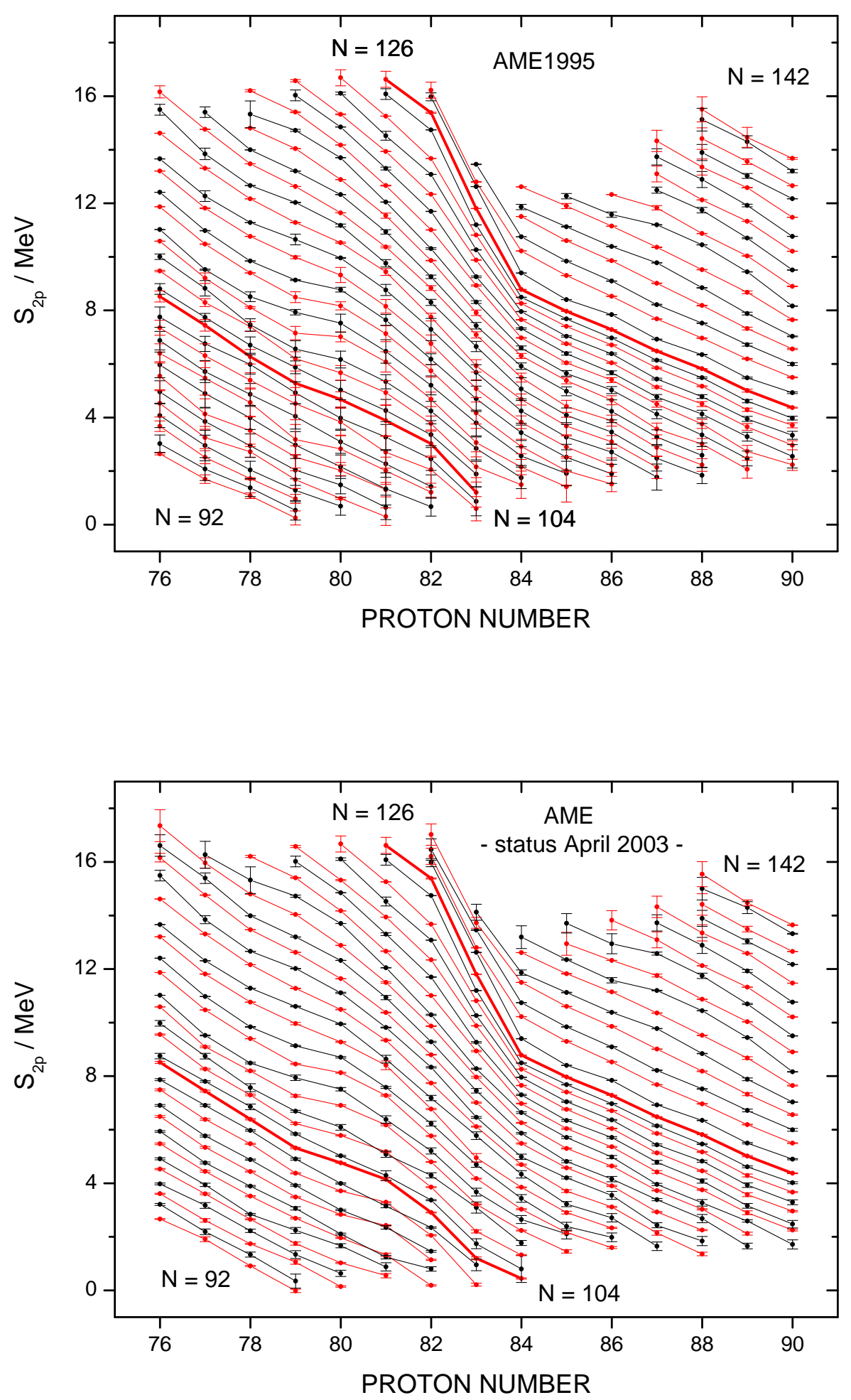

Fig. 14. Comparison of two-proton separation energies $S_{2 p}$ as obtained in AME1995 [50] and 2003. Color figure online. 
In order to investigate this anomalous behavior further, the two-proton separation energies are plotted in Fig. 15 against the neutron number and the isotopes of an element are connected by lines. This presentation shows very clearly in the region of the lead isotopes at $Z=82$ the steadily increasing shell gap from mid-shell $N=104$ towards the magic neutron number $N=126$. Also the above mentioned irregularities in the thallium and (less pronounced) in the mercury isotopes are revealed distinctly as an odd-even effect. The reason for this effect is unclear. Three possible explanations are discussed in the following:

1. The masses of ${ }^{181} \mathrm{Tl}$ and ${ }^{183} \mathrm{Tl}$, determined with ISOLTRAP cyclotron resonances and included in AME2003, were wrongly assigned to the ground states of these isotopes. However, an assignment to the isomeric states (excitation energy $E \geq 800 \mathrm{keV}$ or $E \geq 600 \mathrm{keV}$ ) would increase the staggering even further. Also the mass of ${ }^{187} \mathrm{Tl}$ is determined to more than $60 \%$ by ISOLTRAP, but in this case indirectly via the $Q$ value of the $\alpha$ decay and the directly measured mass of ${ }^{191} \mathrm{Bi}$. Also here, a possible problem in the assignment can be excluded due to the sufficiently high resolving power for separating ground and isomeric states and because of the short half-life of the isomer.

2. Some mass measurements might be wrong. This explanation is contradictory to the fact that the mass values in question stem from different sources and mass spectrometric techniques (ISOLTRAP, ESR, $Q$ values, etc.). Furthermore, the odd-even staggering is not an isolated phenomenon but appears also (less pronounced) in the neighboring isotopic chain of mercury. This is not easily explicable since the mercury and the thallium isotopes are not interconnected by $Q_{\alpha}$ values.

3. The odd-even staggering of the two-proton separation energies in the thallium isotopic chain is factual and represents a nuclear structure effect. This would imply a stronger pairing effect for neutron numbers around mid-shell $(N=104)$ than for nearly double-magic thallium nuclei with $Z=81$ and $N \approx 126$. Similar to the drastic odd-even staggering observed in the charge radii of the mercury isotopes (caused by shape transitions) [58] these phenomena depend very critically on the neutron and proton configurations and very elaborated calculations are required to reproduce these effects. 


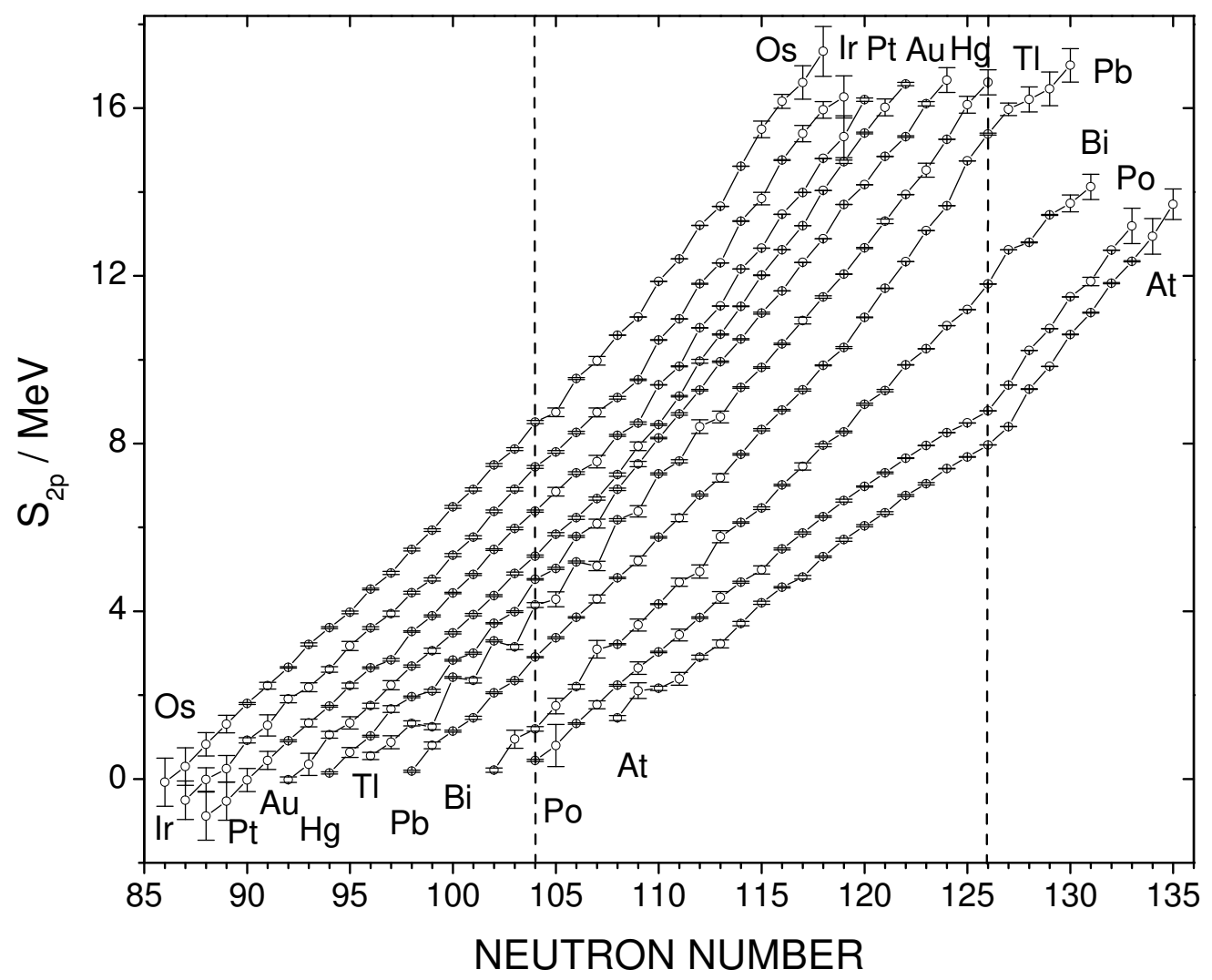

Fig. 15. Two-proton separation energies as a function of neutron number. 


\subsection{Correlation between pairing energies and nuclear charge radii}

Nuclear masses, as determined in this work, and nuclear charge radii, as obtained by optical spectroscopy from isotope shift measurements, illustrate global and collective properties of nuclear matter. The mass is a measure of the binding energy and the charge radii reflect static and dynamic deformations. The nuclear system, as a function of protons and neutrons, maximizes the binding energy according to the nuclear shape. The pairing energy, which is about $1 \mathrm{MeV}$, plays an important role in this context and becomes visible as odd-even effect in the masses as well as in the charge radii.

Due to the complex interplay of single-particle and collective degrees of freedom, one observes in the region of the nuclear chart investigated in this work both a spherical and deformed shape in the same nucleus at low excitation energies. This phenomenon, first observed at ISOLDE/CERN in the seventies by optical spectroscopy [5, 59] is known as "shape coexistence or shape isomerism" and nowadays an extensive bulk of data has been collected throughout the chart of nuclei [8,10,57,58]. Shape coexistence is visible in the vicinities of the shell closures 8, 10,58]. Most clearly it is observed in the slope of charge radii in the mercury isotopic chain: It manifests itself in a huge odd-even shape staggering near the $N=104$ mid-shell region [58] and a large difference in charge radii and therefore nuclear deformation between the ground and isomeric state in ${ }^{185} \mathrm{Hg}$ [6] (Fig. [18). Since the nuclear states of very different configuration are (almost) degenerate in energy, one cannot expect to observe any major trend in the slope of the masses or binding energies as a function of neutron number. In fact, only now the high mass resolving power $m / \Delta m(\mathrm{FWHM})$ of up to $10^{7}$ of the Penning trap mass spectrometer ISOLTRAP enables one to resolve ground and isomeric states in this region of the nuclear chart where shape coexistence occurs. The implementation of many new accurate mass data from ISOLTRAP ([12,13,56] and this work) to the data base of the Atomic Mass Evaluation and a large data set of charge radii [4,60] stimulated an attempt to reveil a correlation between masses and charge radii [61.

In order to uncover finer details in the binding energies regarding odd-even staggering and general trends [62,63], the absolute values of the deduced pairing gap parameters for neutrons are plotted instead of the experimental masses.

The three-point indicator for the shell gap parameter $\Delta$ is given by

$$
\Delta^{3}(N)=\frac{(-1)^{N}}{2}[B(N-1)+B(N+1)-2 B(N)]
$$

which is often interpreted as a measure of the empirical pairing gap 62. Another commonly used relation is the four-point indicator, which averages 
the $\Delta^{3}(N)$ values and is given by

$$
\Delta^{4}(N)=\frac{1}{2}\left[\Delta^{3}(N)+\Delta^{3}(N-1)\right] .
$$

Figures 16- 20 compare the results from mass measurements with those from isotope shift determinations for the isotopic chains of platinum, gold, mercury, thallium, and lead. For these elements, extensive data are available. In order to allow an easy comparison of pairing gap parameters with charge radii for the different elements, the scales of the $\mathrm{x}$ - and $\mathrm{y}$-axis are made identical in Figs. 16- 20. The pairing gap parameters of the isotopic chains of the even- $Z$ elements lead, mercury and platinum are nearly identical in the regions of $N<99$ and $107<N<119$, respectively. The pairing gap parameters for the isotopes of the odd- $Z$ elements thallium and gold show a similar behavior but the pairing gap is smaller in magnitude. Around mid-shell neutron number $N=104$ a minimum develops which is barely visible in the case of lead. It becomes sharper and is shifted to $N=105$ for thallium, distinct and narrow for mercury but very much pronounced and broader for gold and platinum.

The slopes of the isotopic chains of lead, thallium and mercury are almost identical in the region where data are available for the same neutron number. Only the odd-even staggering is much more pronounced for the thallium isotopes as compared to those of lead and mercury.

Down to neutron number $N=108$, the slope of the charge radii for the isotopes of gold and platinum is a little bit smaller than for the other isotopic chains which indicates increasing deformation. For $N=105$ in the case of mercury and $N=107$ for gold a sudden change of the charge radii is observed which is due to a sharp change in deformation.

Comparing the slopes of pairing parameters with those of the charge radii, one observes that the extremely sharp discontinuity at the shell closure at $N=126$ is visible in the charge radii only as a small kink in their slope, that the pronounced increase of deformation at mid-shell $N=104$ is reflected as a local minimum in the pairing parameters, and that the size of the odd-even staggering in the pairing parameter $\Delta^{3}(N)$ is not reflected in the size of the one of the charge radii. There is an interesting observation: For thallium an unusual odd-even staggering between the isomers below mass number $A=193$ is observed.

The new data obtained in this region of the nuclear chart near $Z=82$ will hopefully stimulate theoretical work to develop nuclear models further which are able to describe simultaneously experimental mean square charge radii as well as experimental masses of spherical and deformed nuclei in a satisfactory way. Up to now, only little attention has been paid to such investigations which concern model-independent gross properties of nuclear matter. For example, the charge radii are only poorly described by macroscopic-microscopic mass models which are, however, quite successful in describing nuclear masses 64]. Even more demanding is a description of the odd-even staggering of masses 


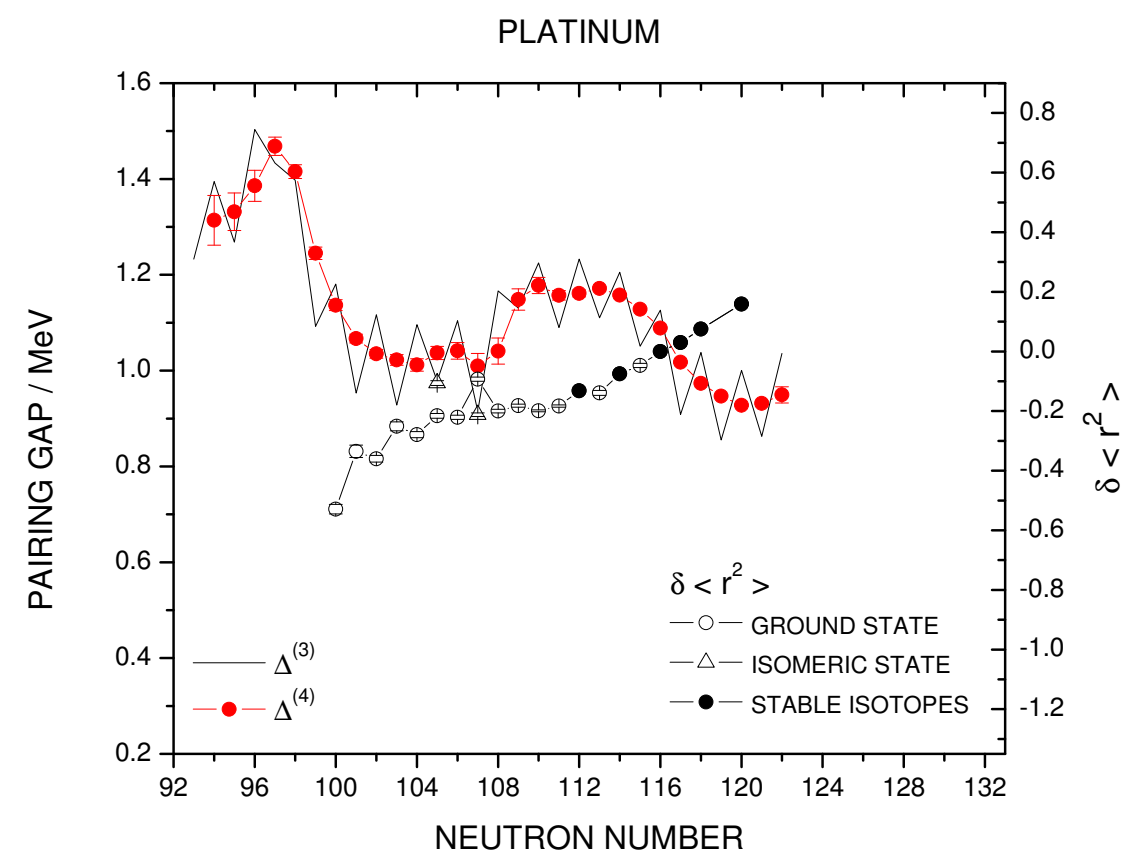

Fig. 16. Comparison of neutron pairing gap energies $\Delta^{(3)}$ and $\Delta^{(4)}$ with nuclear mean square charge radii $\delta<r^{2}>$ for the isotopic chain of platinum. The uncertainties of the $\delta<r^{2}>$ values are smaller than the size of the symbols. Color figure online.

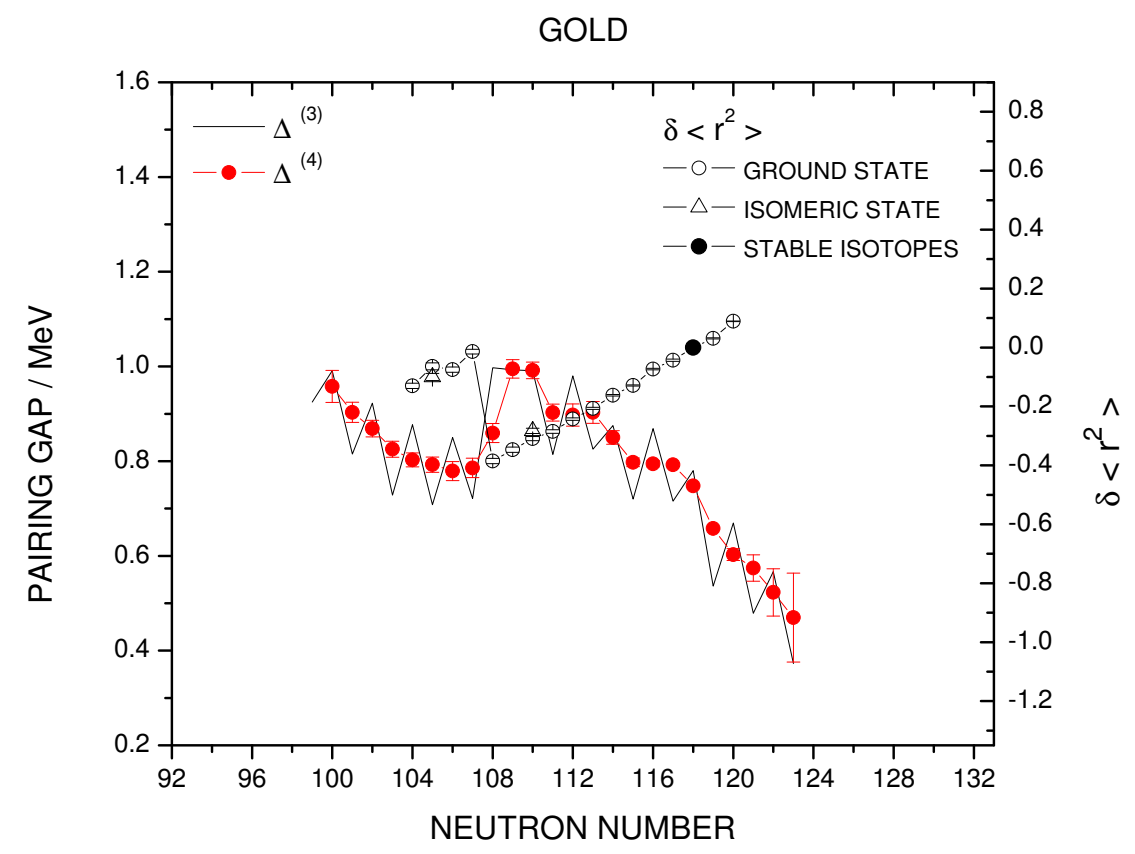

Fig. 17. Same as Fig. 16 but for gold isotopes.

and of the charge radii as attempted in Ref. [65] for spherical nuclei. 


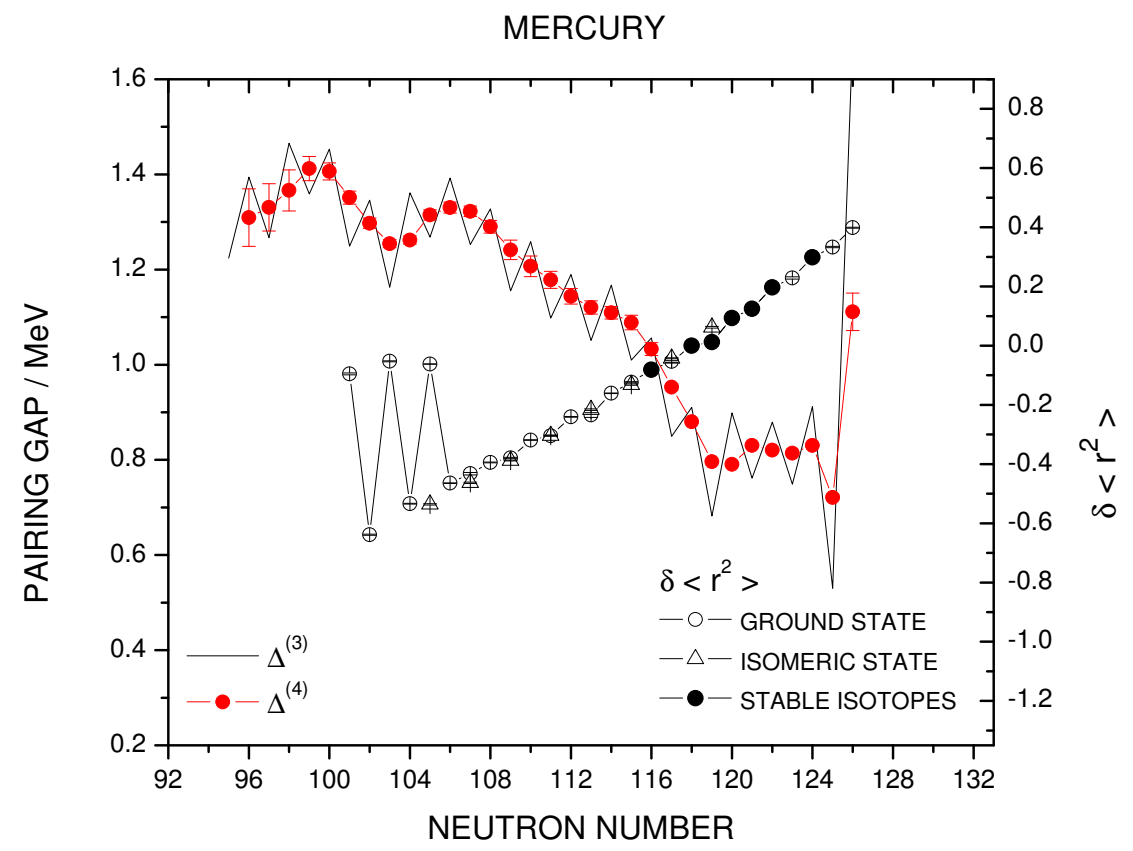

Fig. 18. Same as Fig. 16 but for mercury isotopes.

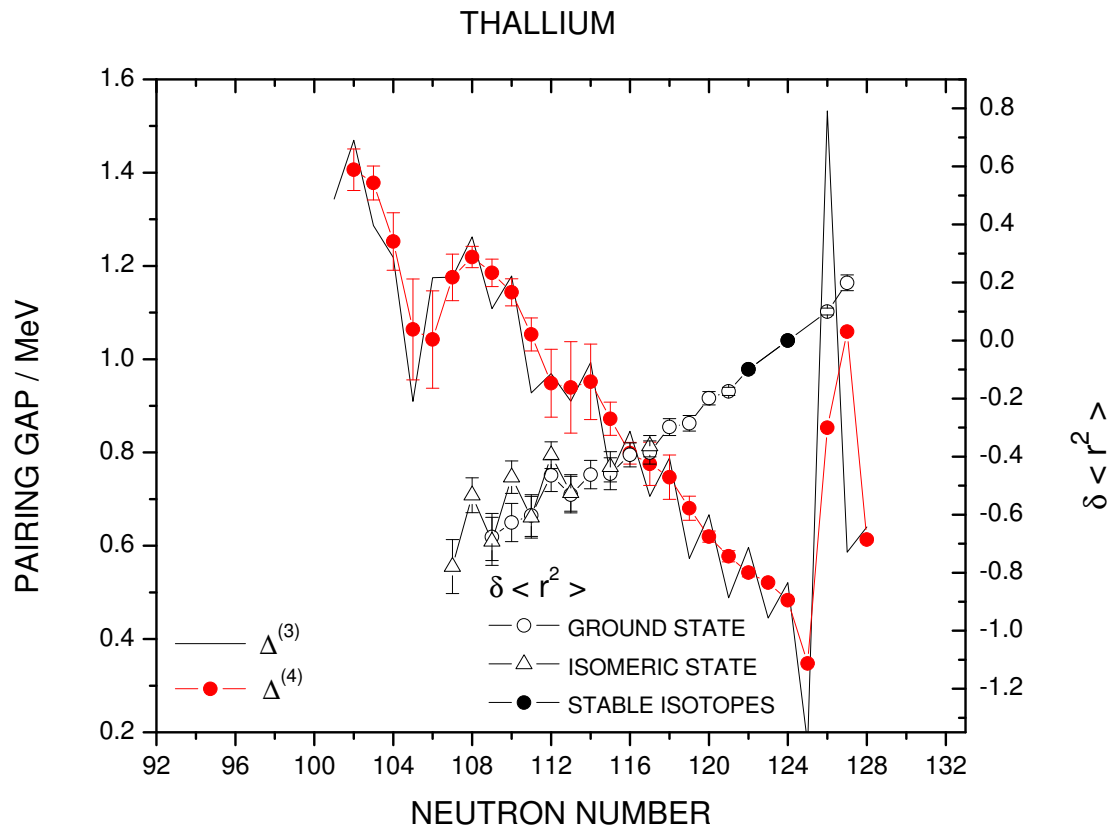

Fig. 19. Same as Fig. 16 but for thallium isotopes. Note that the radii of the isomer exhibit the staggering behavior. 
LEAD

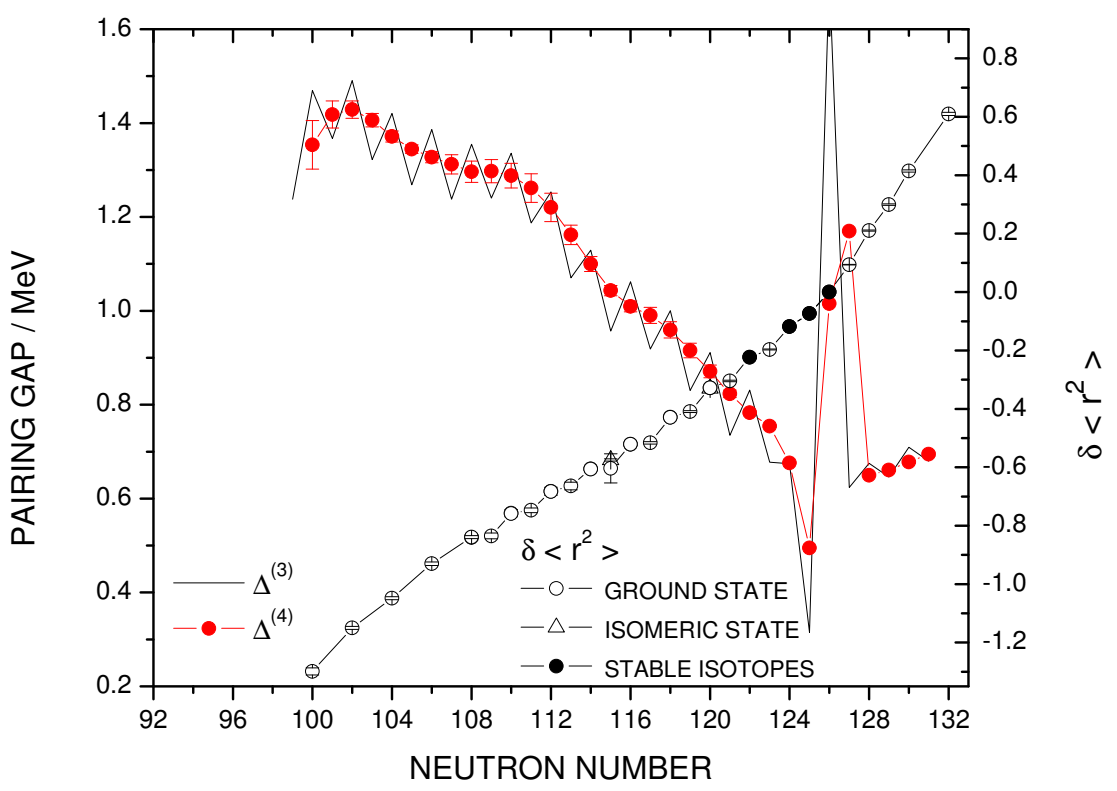

Fig. 20. Same as Fig. 16 but for lead isotopes.

\section{Summary and Outlook}

The fine structure of the mass surface is important for the elucidation of the dramatic shape changes that appear so suddenly in this region of the nuclear chart. For most of the nuclides that were investigated in this work a clear mass-to-state assignment was possible. Few exceptions due to an insufficient resolving power during the measurement are the nuclides ${ }^{187} \mathrm{Tl},{ }^{190} \mathrm{Bi},{ }^{192} \mathrm{Bi}^{m}$, and ${ }^{197} \mathrm{Bi}$. Spectroscopic data giving production ratios could assist these identifications using Eq. (5). The excitation energies of bismuth isotopes (odd-odd nuclei) suffer from particulary high uncertainties. Hence, these cases should be addressed in a future mass measurement requiring the following conditions: (i) If the half-lives of the states are sufficiently long in order to allow an extremely high-resolution measurement: A mass resolution of, for example, $40 \mathrm{keV}$ can be obtained with an excitation time of $10 \mathrm{~s}$. In these cases the count-rate-class analysis can give a meaningful interpretation, i.e. a possible contamination is detected. (ii) Similar to the measurements of both states of ${ }^{187} \mathrm{~Pb}$ [66], a narrow-band laser ionization with a bandwidth of $1.2 \mathrm{GHz}$ can be used to selectively ionize one of the states. As a prerequisite, the element of interest has to be accessible for laser ionization and the hyperfine structure splitting of the isomeric components with different nuclear spin has to be known. (iii) In cases where the half-life is not sufficiently long to perform a measurement with extended excitation time, the mass determination should be supported by nuclear spectroscopy in order to enable a clear identification of states. 


\section{Acknowledgements}

We gratefully acknowledge the ISOLDE technical group and the RILIS group for assistance during the experiments. This work was supported by the German Ministry for Education and Research (BMBF contracts 06MZ962I, 06MZ21S, and 06LM968), by the European Commission (contracts HPRI-CT-1998-00018 (LSF) and HPRI-CT-2001-50034 (NIPNET)), and by the Association of Helmholtz Research Centers (contract VH-NG-037).

\section{References}

[1] D. Lunney, J.M. Pearson, and C. Thibault, Rev. Mod. Phys. 75 (2003) 1021.

[2] K. Blaum, Phys. Rep. 425 (2006) 1.

[3] E.W. Otten, Treatise on Heavy Ion Physics 8 (1989) 517, and references therein.

[4] H.-J. Kluge and W. Nörtershäuser, Spectrochim. Acta B 58 (2003) 1031, and references therein.

[5] J. Bonn et al., Phys. Lett. B 38 (1972) 308.

[6] P. Dabkiewicz et al., Phys. Lett. B 82 (1979) 199.

[7] S. Frauendorf and V.V. Pashkevich, Phys. Lett. 55B (1975) 365.

[8] J.L. Wood et al., Phys. Rep. 215 (1992) 101.

[9] A.N. Andreyev et al., Nature 405 (2000) 430.

[10] K. Heyde et al., Phys. Rep. 102 (1983) 291.

[11] K. Heyde, Nucl. Phys. A. 507 (1990) 149c.

[12] S. Schwarz, Ph.D. thesis, University of Mainz (1998), ISBN 3-8288-0735-6.

[13] S. Schwarz et al., Nucl. Phys. A 693 (2001) 533.

[14] R. Fossion et al., Nucl. Phys. A 697 (2002) 703.

[15] E. Coenen et al., Phys. Rev. Lett. 54 (1985) 1783.

[16] P. Van Duppen et al., Nucl. Phys. A 529 (1991) 268.

[17] G. Bollen et al., J. Mod. Opt. 39 (1992) 257.

[18] F. Herfurth et al., Eur. Phys. J. A 25 (2005) 17.

[19] T. Radon et al., Nucl. Phys. A 677 (2000) 75.

[20] Yu.A. Litvinov, Ph.D. thesis, University of Giessen (2003). 
[21] Yu.A. Litvinov et al., Nucl. Phys. A 756 (2005) 3.

[22] M. Epherre et al., Phys. Rev. C 19 (1979) 1504.

[23] G. Audi et al., Nucl. Phys. A 378 (1982) 443.

[24] Z. Patyk et al., Nucl. Phys. A 491 (1989) 267.

[25] G. Bollen et al., Nucl. Instr. Meth. A 368 (1996) 675.

[26] F. Herfurth et al., Nucl. Instr. Meth. A 469 (2001) 254.

[27] M. Mukherjee et al., submitted to Eur. Phys. J. A.

[28] E. Kugler, Hyp. Int. 129 (2000) 23.

[29] V.I. Mishin et al., Nucl. Instr. Meth. B 73 (1993) 550.

[30] U. Köster et al., Spectrochim. Acta 58 (2003) 1047.

[31] H. Raimbault-Hartmann et al., Nucl. Instr. Meth. B 126 (1997) 378.

[32] G. Savard et al., Phys. Lett. A 158 (1991) 247.

[33] G. Gräff, H. Kalinowski, and J. Traut, Z. Phys. A 297 (1980) 35.

[34] M. König et al., Int. J. Mass. Spectrom. Ion Process. 142 (1995) 95.

[35] G. Bollen, Nucl. Phys. A 693 (2001) 3.

[36] G. Sikler et al., Nucl. Phys. A 763 (2005) 45.

[37] A. Kellerbauer et al., Eur. Phys. J. D 22 (2003) 53.

[38] K. Blaum et al., J. Phys. B 36 (2003) 921.

[39] S. George et al., Phys. Rev. Lett. 98 (2007) 162501.

[40] C. Yazidjian et al., Phys. Rev. C 76 (2007) 024308.

[41] C. Guénaut et al., Phys. Rev. C 75 (2007) 044303.

[42] P. Delahaye et al., Phys. Rev. C 74 (2006) 034331.

[43] K. Blaum et al., Eur. Phys. J. A 15 (2002) 245.

[44] M. König, Diploma thesis, University of Mainz (1991).

[45] G. Bollen et al., Phys. Rev. C 46 (1992) R2140.

[46] D. Beck et al., Nucl. Instr. Meth. B 126 (1997) 374.

[47] G. Audi et al., Nucl. Phys. A 624 (1997) 1.

[48] G. Audi, compilation of an intermediate atomic mass evaluation in 2002. This evaluation did not contain the ISOLTRAP data presented in this work but all other mass data published up to this date. IAME is used for comparison with our mass values, since those are already included in the AME 2003 [52]. 
[49] M.P. Bradley et al., Phys. Rev. Lett. 83 (1999) 4510.

[50] G. Audi and A.H. Wapstra, Nucl. Phys. A 595 (1995) 409.

[51] G. Audi and A.H. Wapstra, Nucl. Phys. A 565 (1993) 1.

[52] G. Audi, A.H. Wapstra, and C. Thibault, Nucl. Phys. A 729 (2003) 337.

[53] G. Audi, Hyp. Int. 132 (2001) 7.

[54] A.H. Wapstra, G. Audi and C. Thibault, Nucl. Phys. A 729 (2003) 129.

[55] D. Beck et al., Eur. Phys. J. A 8 (2000) 307.

[56] A. Kohl, Ph.D. thesis, University of Heidelberg (1999).

[57] R. Julin et al., J. Phys. G 27 (2001) R109.

[58] G. Ulm et al., Z. Phys. A 325 (1986) 247.

[59] J. Bonn et al., Phys. Lett. B 36 (1971) 41.

[60] H. De Witte et al., Phys. Rev. Lett. 98 (2007) 112502.

[61] C. Weber et al., Eur. Phys. J. A 25 Supplem. 1 (2005) 201.

[62] W. Satula et al., Phys. Rev. Lett. 81 (1998) 3599.

[63] J. Dobaczewski et al., Phys. Rev. 63 (2001) 024308.

[64] F. Buchinger et al., Phys. Rev. C 72 (2005) 057305.

[65] S.A. Fayans and D. Zawischa, Int. J. Modern Phys. B 15 (2001) 1684.

[66] C. Weber et al., Phys. Lett. A 347 (2005) 81. 